\title{
POLYMOLECULARITY AND POLYDISPERSITY IN MOLECULAR WEIGHT DETERMINATIONS
}

\author{
HAN.S-GeORg ELIAS
}

Midland Macromolecular Institute, Midland, Mich. 48640, USA

\begin{abstract}
The influence of polymolecularity on molecular weight determinations is reviewed. Several molecular weight methods give so-called mixed averages, i.e. averages which are based on more than one moment of the molecular weight distribution or on powers of the moment. Molecular weight averages can be subdivided into simple, exponential resolved, order resolved and power resolved averages. Mixed averages depend on the shape of the solute and its interaction with the solvent. Simple averages of molecular weight can be calculated from combinations of averages of different properties with the help of the $\Pi$-theorem. A related problem is that of the so-called polymolecularity corrections. New experiments show a definite influence of the type and the width of the molecular weight distribution on the second osmotic virial coefficients which is not predicted by present theories. Methods are given to distinguish between open, closed, molecule-based and segment-based associations. The influence of the polymolecularity of unimers on the polydispersity of the resulting multimers is discussed for several types of association. In general, a sharpening effect is observed. Equations are given which may help detect polydispersity in open and closed association. Debye's treatment of micellar solutions cannot be used to determine premicellar equilibria.
\end{abstract}

\section{INTRODUCTION}

All synthetic and many biological polymers exhibit a more or less broad molecular weight distribution (molar mass distribution) ${ }^{1}$, which influences many properties of macromolecular materials. Although the effects of this distribution have to be eliminated first before conclusions can be drawn on the effect of other quantities (such as composition, configurational statistics, conformational statistics, supermolecular structures, etc.) on certain properties, astonishingly little work has been done to quantitatively evaluate the effect of the molecular weight distribution on the various properties $^{2}$. This is even true for the methods which are most directly affected by the molecular weight distribution, namely molecular weight determinations.

Molecular weight determinations are normally carried out by measuring certain molecular weight dependent properties at various concentrations. Typical molecular weight dependent properties are, e.g., the osmotic pressure, the Rayleigh ratio, the sedimentation coefficient, etc. Because the numerical values of these properties are influenced by various interactions, the properties have to be extrapolated to zero concentration. The extrapolation 
procedures require theoretical guidelines, i.e. they require a molecular, thermodynamic, hydrodynamic, etc., model of the solution.

It has been long known from statistical thermodynamics that the concentration dependence of the chemical potential of the solvent (and consequently the osmotic pressure) of solutions of non-electrolytes can be expressed as a power series with positive integers of the solute concentration ${ }^{3}$. Because of the relationship between chemical potential and molecular weight, a similar power series describes the concentration dependence of apparent molecular weights. This power series can be generalized to

$$
\left(M_{g}\right)_{\mathrm{app}}^{-1}=M_{g}^{-1}+\alpha\left(A_{2}\right)_{\mathrm{av}} c+\beta\left(A_{3}\right)_{\mathrm{av}} c^{2}+\ldots
$$

$M_{g}$ is the $g$-average of the molecular weight, e.g. the number average $(g=n)$, the weight average $(g=w)$, etc. The apparent $g$-average molecular weight $\left(M_{g}\right)_{\text {app }}$ is the molecular weight calculated from experimental data at finite concentrations $c$ with the help of an equation valid for zero concentration only. The second, third ... virial coefficients $\left(A_{2}\right)_{\mathrm{av}},\left(A_{3}\right)_{\mathrm{av}} \ldots$ represent different averages if different methods are employed. For colligative methods, one gets ${ }^{4}$

$$
\left(A_{2}\right)_{\mathrm{av}}^{\mathrm{OP}}=\sum_{i} \sum_{j} w_{i} w_{j}\left(A_{2}\right)_{i j}
$$

and for weight average methods (light scattering, equilibrium ultracentrifugation $)^{4-6}$

$$
\left(A_{2}\right)_{\mathrm{av}}^{\mathrm{LS}}=\left(\sum_{i} \sum_{j} w_{i} w_{j} M_{i} M_{j}\left(A_{2}\right)_{i j}\right) M_{w}^{-2}
$$

where $w_{i}, w_{j}=$ mass fractions of species $i$ and $j$, respectively. The coefficients $\alpha$ and $\beta$ are also method dependent, giving $\alpha=1$ for osmotic pressure measurements, $\alpha=2$ for light scattering experiments and $\alpha=2 f(\xi)$ for sedimentation equilibrium experiments ${ }^{7}$ where

$$
\begin{aligned}
f(\xi)=1+\left[\xi M_{z}^{2} / 12\right]+\left[M_{z+1} / M_{z}\right)^{2} & \\
& \left.-2\left(M_{z+1} M_{z+2}\right)^{2} / M_{z}^{4}\right]\left(\xi M_{z}\right)^{4} / 720+\ldots
\end{aligned}
$$

The increase of the amount of molecules is directly given by the increase of the mass of solute in non-associating non-electrolyte solutions. This is no longer true for associating solutes because less than a proportional number of particles are formed by an increase of the mass concentration. The relationship between mass and mole concentrations obviously depends on the type of association. For all practical purposes, the extent of the association can be considered independent of the type and magnitude of the other interactions, e.g. solute/solvent interactions and/or intramolecular associations. In many cases the interactions are such that the association can be assumed to happen in a state similar to a theta state. We can thus write

$$
\left(M_{g}\right)_{\mathrm{app}}^{-1}=\left(M_{g}\right)_{\mathrm{app}, \theta}^{-1}+\left(\sum_{i} \sum_{j} c_{i} c_{j}\left(A_{2}\right)_{i j} / c^{2}\right) c+\ldots
$$

where $\left(M_{g}\right)_{\mathrm{app}, \boldsymbol{\theta}}$ is the concentration dependent association term. 
This paper will discuss the influence of polymolecularity on the type and magnitude of the molecular weight averages $M_{g}$ (see equation 1), on the magnitudes of the different second virial coefficients (see equations 2 and 3), and on the association of molecules (see equation 5), especially on the polydispersity of the resulting associates (aggregates, micelles, etc.). The effects of polymolecularity on associated solutes are treated for theta systems only, because the problem of the virial term in associating systems is insurmountable at the present time. The approach will be mainly mathematical and based on certain physically most likely models, because this will allow a unified treatment. Little attempt is made to compare the mathematical predictions with experimental findings, mainly because the few, if any, data reported in literature are mostly incomplete for our purposes.

The problem of molecular weight and other averages has been recently reviewed ${ }^{2}$. Association processes have been discussed in general ${ }^{8,9}$, and with special emphasis on protein $s^{10,11}$, synthetic polymers ${ }^{12}$, sedimentation equilibrium ${ }^{13}$ and light scattering ${ }^{9}$. None of the review articles treats the effects of polymolecularity and polydispersity adequately, if at all.

\section{GENERAL ASPECTS OF POLYMOLECULARITY}

\section{Types of averages}

The distribution function of any property $E$ can be described by a sufficient number of moments. Moments $\mu$ of the $g$-distributions of the properties $E$ with respect to the origin are defined via

$$
\mu_{g}^{(q)}(E) \equiv \sum_{i} G_{i} E_{i}^{q}
$$

where $q=$ order of the moment and

$$
G_{i} \equiv g_{i} /{ }_{i} \sum g_{i}=g_{i} / g
$$

is the normalized statistical weight of the species $i$. The physical unit of a moment of a property $E$ thus depends not only on the physical unit of the property but also on the order of the moment. The order may take any value, i.e. it may be a rational or irrational number.

An average, on the other hand, is defined in such a manner that the physical unit of the average is always identical with the physical unit of the property. This requirement is of course met by a number of expressions. Hence, there are a number of classes of property averages, e.g. simple averages, exponential resolved averages, order resolved averages, etc. In each class, number, weight, length, area, etc., averages may be defined. The following discussion is restricted to molecular weight averages and moments.

Simple averages represent the first moment of the molecular weight distribution curve:

$$
M_{g}=\sum_{i} G_{i} M_{i}=\mu_{g}^{(1)}(M)
$$

They are always arithmetic averages. Most familiar are the number $\left(G_{i}=x_{i}=\right.$ mole fraction), weight $\left(G_{i}=w_{i}=\right.$ mass fraction $)$ and $z$-averages $\left(G_{i}=Z_{i}=w_{i} M_{i}\right)$. 
Exponential resolved averages are also based on a single moment. Because the order of this moment is different from one, the whole moment has to be taken to the inverse power of the order of the moment to yield an average

$$
M_{\mathrm{av}}=\left(\sum_{i} G_{i} M_{i}^{q}\right)^{1 / q}=\left(\mu_{g}^{(q)}\right)^{1 / q}
$$

Exponential resolved averages are normally named after the method, i.e. one speaks of viscosity average molecular weight, sedimentation averages, diffusion averages, etc. This nomenclature is, however, not sufficient because the average given by a certain method may also depend on the method of evaluation and thus on the statistical weight used by the evaluation procedure. The well-known so-called viscosity average molecular weight (with $q=a_{\eta}=$ exponent in the intrinsic viscosity/molecular weight relationship, $[\eta]=K_{\eta} M^{a_{\eta}}$ ) is thus more properly called a mass (or weight) average exponential resolved average because intrinsic viscosities of mixtures of polymers without specific interactions are always mass averages ${ }^{14-16}$.

Order resolved averages are composed of two moments of the same statistical weights which differ in their orders by one:

$$
M_{\mathrm{av}}=\sum_{i} G_{i} M_{i}^{q+1} / \sum_{i} G_{i} M_{i}^{q}=\mu_{g}^{(q+1)} / \mu_{g}^{(q)}
$$

Order resolved averages are quite frequent if molecular weights are determined by the Svedberg method (combination of sedimentation and diffusion coefficients) (see also below). The molecular weight from gel permeation chromatography is also an order resolved average $\left(G=w \text { and } q=a_{\eta}\right)^{17}$.

Geometric averages are normally classified as the square root of the product of two simple molecular weights with different statistical weights $g$ and $h$ :

$$
M_{\mathrm{av}} \equiv\left(M_{g} M_{h}\right)^{\frac{1}{2}}
$$

An example is the so-called $M_{-1}$ average ${ }^{18}$, which has been shown to be a geometric average (see reference 2):

$$
M_{-1} \equiv\left(\sum_{i} m_{i} / \sum_{i} m_{i} M_{i}^{-2}\right)^{\frac{1}{2}}=\left(M_{n} M_{n-1}\right)^{\frac{1}{2}}
$$

where $m_{i}=$ mass of species $i$. A closer inspection shows that the geometric averages are a sub-class of the exponential resolved averages. Equation (12) can be written as

$$
M_{-1}=\left(\sum_{i}^{\cdot} w_{i} M_{i}^{-2}\right)^{-\frac{1}{2}}
$$

It is thus the exponential resolved weight average of the minus second moment of the molecular weight. Another example is the 'root mean square' average ${ }^{19}$, which is nothing but the exponential resolved weight average of the second moment of the molecular weight ${ }^{2}$ :

$$
M_{\mathrm{rms}}=\left(M_{z} M_{w}\right)^{\frac{1}{2}}=\left(\sum_{i} w_{i} M_{i}^{2}\right)^{\frac{1}{2}}
$$


Power resolved averages are products of moments defined ${ }^{2}$ in such a manner that the exponents of the moments differ by one:

$$
M_{\mathrm{av}}=\left(\sum_{i} G_{i} M_{i}\right)^{p}\left(\sum_{i} G_{i} M_{i}^{-1}\right)^{p-1}=\left(M_{g}\right)^{p}\left(M_{g-1}\right)^{1-p}
$$

Many other averages can be defined on this basis. They all can be covered by a general expression ${ }^{20}$

$$
M_{\mathrm{av}}=\left(\sum_{i} G_{i} M_{i}^{p+q-1} / \sum_{i} G_{i} M_{i}^{q-1}\right)^{1 / p}
$$

There are, however, a variety of averages which cannot be expressed by simple product sums. Sedimentation equilibrium measurements in a density gradient yield, e.g., molecular weight averages which contain exponential expressions $^{21,22}$ :

$$
M_{\sigma}=\frac{\sum_{i} G_{i} M_{i}^{\frac{5}{2}} \exp \left[\left(-M_{i} /\left(2 M_{\sigma}\right)\right]\right.}{\sum_{i} G_{i} M_{i}^{\frac{3}{2}} \exp \left[\left(-M_{i} /\left(2 M_{\sigma}\right)\right]\right.}
$$

where $M_{\sigma}$ is obtained from the half-width of the density distribution.

\section{Statistical weights}

The most commonly used statistical weights can be subdivided into two classes: entity-based and shape-based statistical weights. The class of entitybased statistical weights comprises the number statistical weight, the mass statistical weight, their higher and lower homologues (see below), and their equivalents. The class of shape-based statistical weight consists of the length, area and volume statistical weights.

The class of entity-based statistical weights can be considered as derivatives of the numbers $N_{i}$ of the species $i$ or, of course, their amounts $n_{i}$ or their mole fractions $x_{i}$ :

$$
\begin{gathered}
n_{i}=N_{i} / N_{\mathrm{A}} \\
x_{i}=n_{i} / \sum_{i} n_{i}=N_{i} / \sum_{i} N_{i}=N_{i} / N
\end{gathered}
$$

where $N_{\mathrm{A}}$ is Avogadro's number and $N$ is the total number of molecules. The next higher homologue is the mass

$$
m_{i}=n_{i} M_{i}=N_{i} M_{i} / N_{\mathrm{A}}
$$

or its equivalents such as the mass fraction $w_{i}$ or the so-called weight concentration (volume related mass):

$$
\begin{gathered}
w_{i}=m_{i} / \sum_{i} m_{i}=c_{i} / \sum_{i} c_{i}=c_{i} / c \\
c_{i}=m_{i} / V_{\text {solution }}
\end{gathered}
$$

All other entity related statistical weights can be introduced by analogy with equation (20), i.e. as products of the statistical weight of next lower rank with molecular weight. Examples are 


$$
\begin{aligned}
& (n-1) \text {-statistical wt: } \quad(n-1)_{i}=n_{i} M_{i}^{-1}=m_{i} M_{i}^{-2}=(m-2)_{i} \\
& \text { number statistical wt: } \quad n_{i}=n_{i} M_{i}^{0}=m_{i} M_{i}^{-1}=(m-1)_{i} \\
& \text { mass ('weight') statistical wt: } m_{i}=n_{i} M_{i}=m_{i} M_{i}^{0}=m_{i} \\
& z \text {-statistical wt: } \\
& z_{i}=n_{i} M_{i}^{2}=m_{i} M_{i}=(m+1)_{i} \\
& (z+1)_{i} \text { statistical wt: } \quad(z+1)_{i}=n_{i} M_{i}^{3}=m_{i} M_{i}^{2}=(m+2)_{i}
\end{aligned}
$$

The first expressions give the commonly used symbols for the different statistical weights; note that these are symbols and not mathematical operations. The second expressions relate amounts and molecular weights, the third expressions mass and molecular weight. The fourth expressions give alternative symbols. One may see that the mass can be taken instead of the amount as the base unit for entity-based statistical weights, or even the $z$-unit, etc.

If the species $i$ are not molecularly homogeneous but exhibit a molecular weight distribution themselves, the molecular weights to be used in equations $(23)-(27)$ should always correspond to the multiplying statistical weight ${ }^{23}$. An example is

$$
(z+1)_{i}=z_{i}\left(M_{i}\right)_{z}=m_{i}\left(M_{i}\right)_{w}\left(M_{i}\right)_{z}=n_{i}\left(M_{i}\right)_{n}\left(M_{i}\right)_{w}\left(M_{i}\right)_{z} \text { etc. }
$$

It must be mentioned that this statement can be generalized for any average of any property ${ }^{24}$.

Shape-based statistical weights are lengths, areas and volumes or the corresponding fractions. They are also related to moles and masses in the case of simple bodies. The interrelationship between entity- and shapebased statistical weights depends, however, on the shape of the species. For spheres, rods (with length $L$ and constant and negligible radius $R$ ) and discs (with constant and negligible height $H$ ) with the surface $A$ one gets, e.g. for the different masses if the densities $\rho_{i}$ are constant:

$$
\begin{array}{ll}
\text { spheres: } & m_{i}=N_{i} V_{i} \rho=N_{i}\left(4 \pi R_{i}^{3} / 3\right) \rho=N_{i}(\rho / 3) R_{i} A_{i} \\
\text { rods: } & m_{i}=N_{i} V_{i} \rho=N_{i}\left(\pi R^{2} L_{i}\right) \rho \approx N_{i}(\rho / 2) R A_{i} \\
\text { discs: } & m_{i}=N_{i} V_{i} \rho=N_{i}\left(\pi R_{i}^{2} H\right) \rho \approx N_{i}(\rho / 2) H A_{i}
\end{array}
$$

\section{Polymolecularity parameters}

The ratio of any two different molecular weight averages or any two different moments of the molecular weight distribution can be used to characterize the width of a molecular weight distribution. The two quantities have to be chosen in such a way that their ratio is a sensitive and absolute measure for the width of the distribution.

In many cases, the ratio of two simple molecular weight averages is a sensitive enough quantity for the characterization of the polymolecularity, e.g. the polymolecularity index,

$$
Q_{w, n}=M_{w} / M_{n}
$$

or its equivalent, the inhomogeneity parameter ${ }^{25}$,

$$
U_{w, n}=\left(M_{w} / M_{n}\right)-1
$$


It can be easily shown that most other polymolecularity parameters such as the ratio of two viscosity averages ${ }^{26}$ or the ratio of two different molecular weight averages from combined sedimentation and diffusion ${ }^{27}$ are, in general, less sensitive than the polymolecularity index or the inhomogeneity parameter (see reference 2).

The standard deviation of the molecular weight distribution can be used as a measure of the polymolecularity ${ }^{28-35}$. As one can see from, e.g., the number standard deviation

$$
\sigma_{n}=\left(M_{w} M_{n}-M_{n}^{2}\right)^{\frac{1}{2}}=\left(U_{w, n}\right)^{\frac{1}{2}} M_{n}
$$

it is more sensitive than the polymolecularity index. The standard deviations are absolute measures for the width of Gaussian distributions, since the quantity $\left(M_{n} \pm \sigma_{n}\right)$ always denotes that 68.26 per cent of the molecular weights are in this range and the quantity $\left(M_{n} \pm 2 \sigma_{n}\right)$ always denotes 95.44 per cent of the molecular weights, etc., independent of the position and the width of the Gaussian distribution curve. The standard deviation is, however, not an absolute measure for the width of other types of distribution, because the fraction of the molecular weight as characterized by $\sigma_{n}$ and its multiples is always a function of the width of the distribution ${ }^{36}$. No attempts have been made so far to introduce absolute measures for the width of distribution curves for other than Gaussian distributions.

\section{MIXED AVERAGES}

\section{Types of molecular weight}

A mixed average will be defined as an average which is not a simple average as defined by equation (9). All mixed averages are thus characterized by the existence of at least one moment with the order $q \neq 1$. Typical examples are the molecular weight determinations from intrinsic viscosity/ molecular weight relationships:

$$
[\eta]=K_{\eta} M^{a_{\eta}}
$$

or from sedimentation and diffusion coefficients via the Svedberg equation:

$$
M_{\mathrm{av}}=\frac{f_{s}}{f_{D}} \frac{R T}{\left(1-\tilde{v}_{2} \rho_{1}\right)} \frac{s}{D}
$$

Other equations leading to mixed averages $M_{\mathrm{av}}$ of molecular weight are summarized in Table 1. Most property/molecular weight relationships can be expressed by exponential equations similar to equation (35) for the whole molecular weight range of interest:

$$
E=K_{E} M^{a_{E}} \quad(E=[\eta], s, D, \text { etc. })
$$

A theoretical justification is lacking in most cases, however. Exponential relationships can be even used if these equations contain additive terms ${ }^{60-63}$, or if the exponents $a_{E}$ are molecular weight dependent themselves ${ }^{64,65}$.

Analysis of the physical units (i.e. 'dimensional analysis') shows that these exponents are related to each other by the so-called exponent rule eno, 61,66-68 $^{50}$

$$
a_{\eta}=2-3 a_{s}=-\left(1+3 a_{\mathrm{D}}\right)
$$



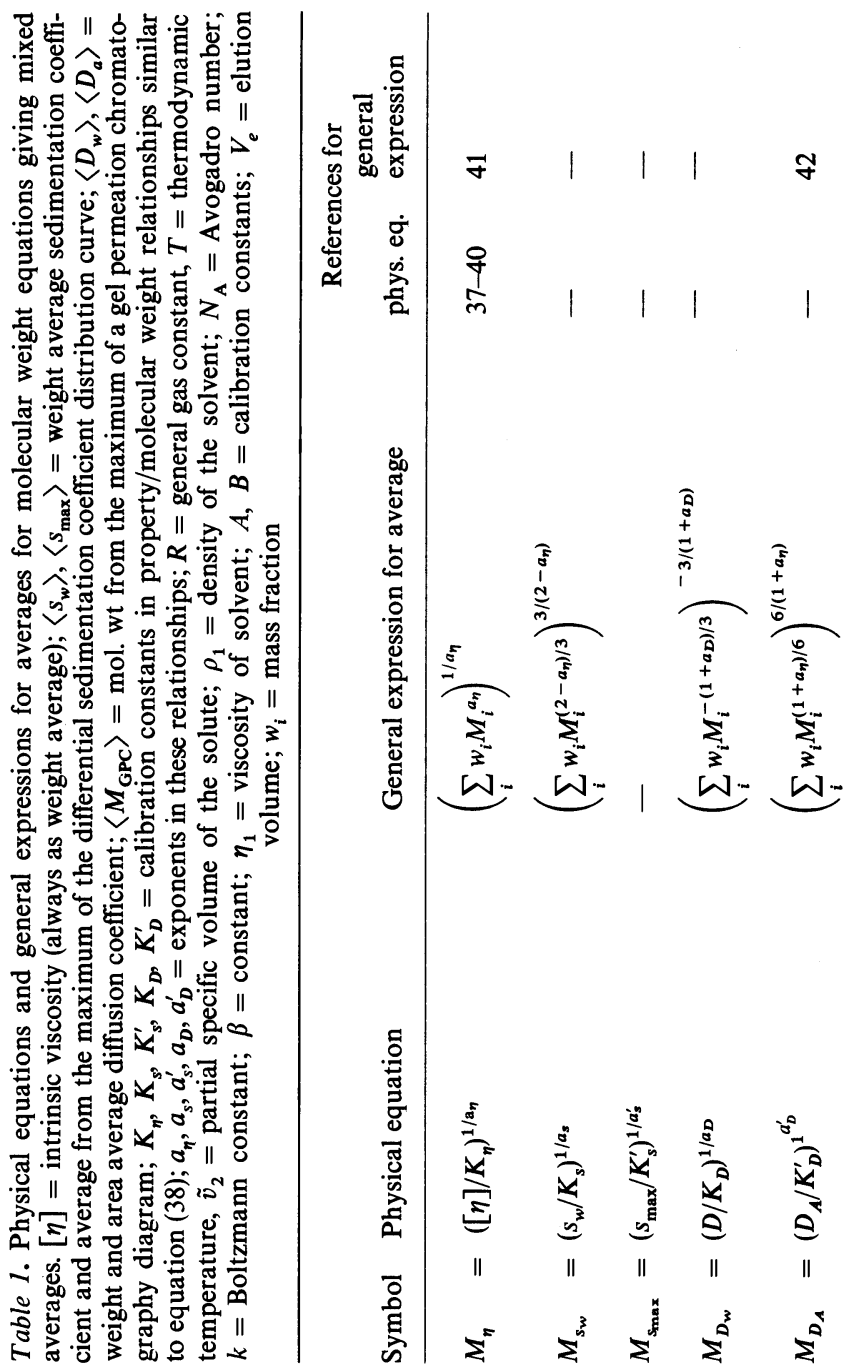
POLYMOLECULARITY AND POLYDISPERSITY

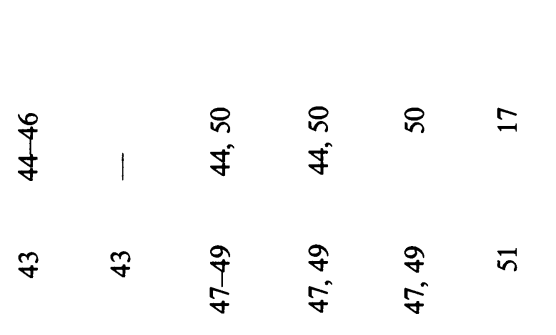

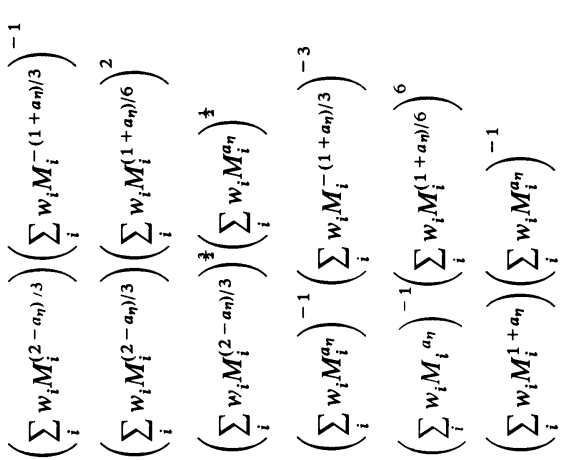

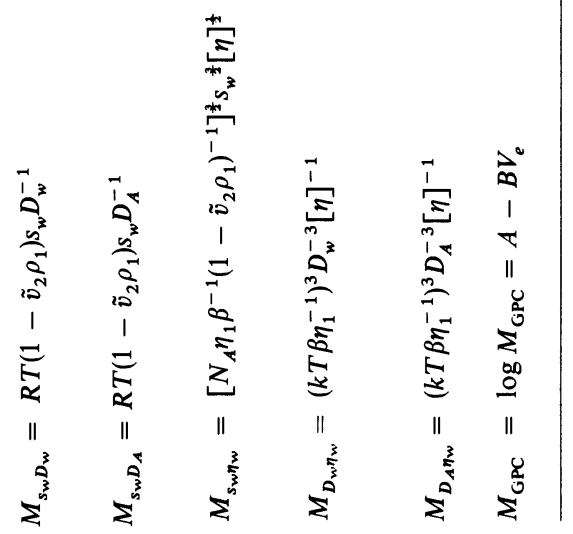




\section{HANS-GEORG ELIAS}

品

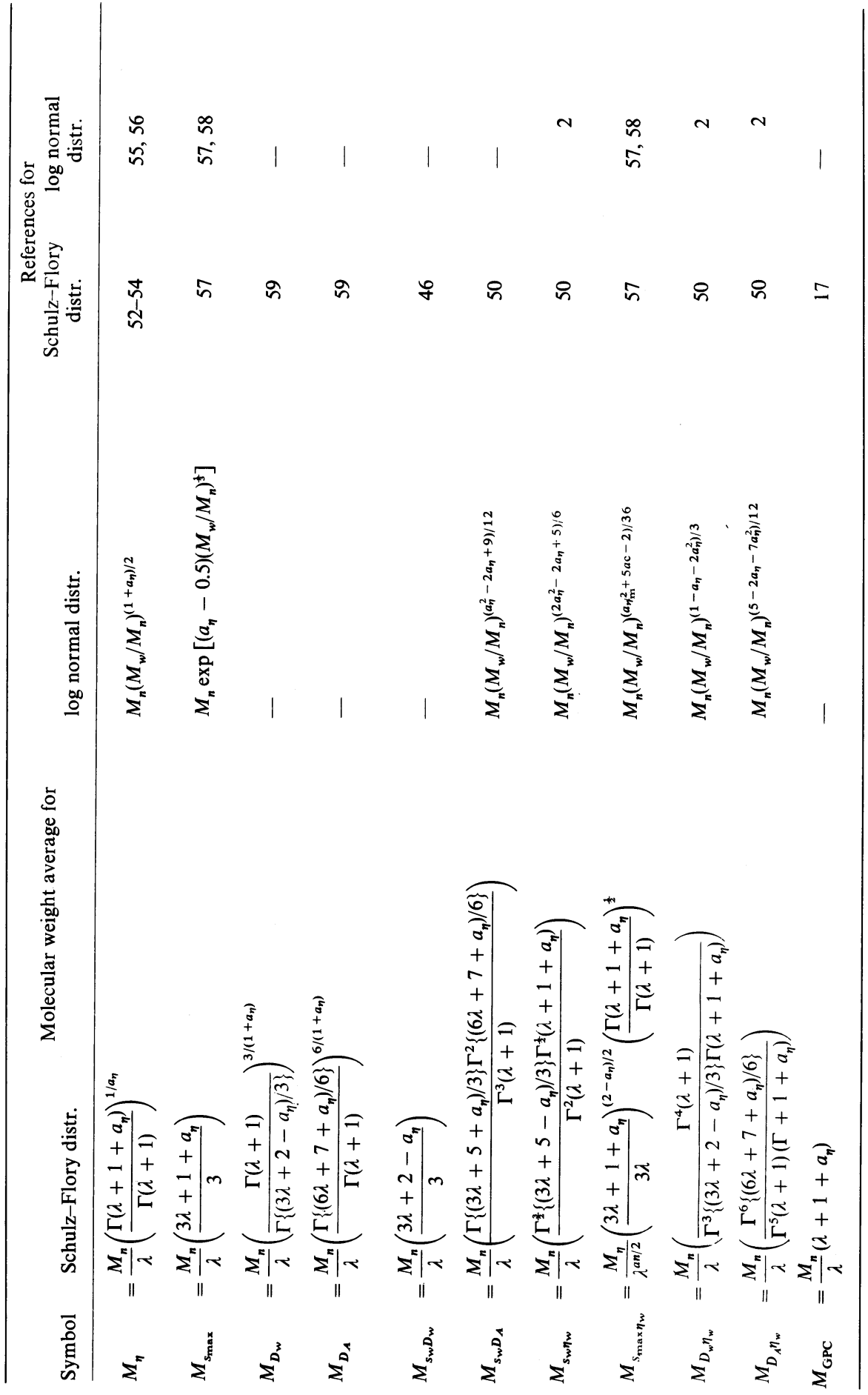


Equations (37) and (38) allow an analysis of the types of molecular weight averages of physical equations typically leading to mixed averages. Examples are equations (35) and (36); others can be found in Table 1. One simply replaces the molecular weight dependent physical quantities like the intrinsic viscosity, the sedimentation coefficient, etc., by their averages (e.g. weight average), expresses the properties of the species $i$ by the corresponding expressions for their molecular weight dependence (see, e.g., equation 37), and finally replaces the individual exponents $a_{D}, a_{s}$, etc., by the exponent $a_{\eta}$ with the help of equation (38). The resulting expressions are then converted into averages or moments. Some of these expressions are summarized in Table 1. They can be replaced by specific functions if the type of the molecular weight distribution is known (Table 2). The resulting molecular weight

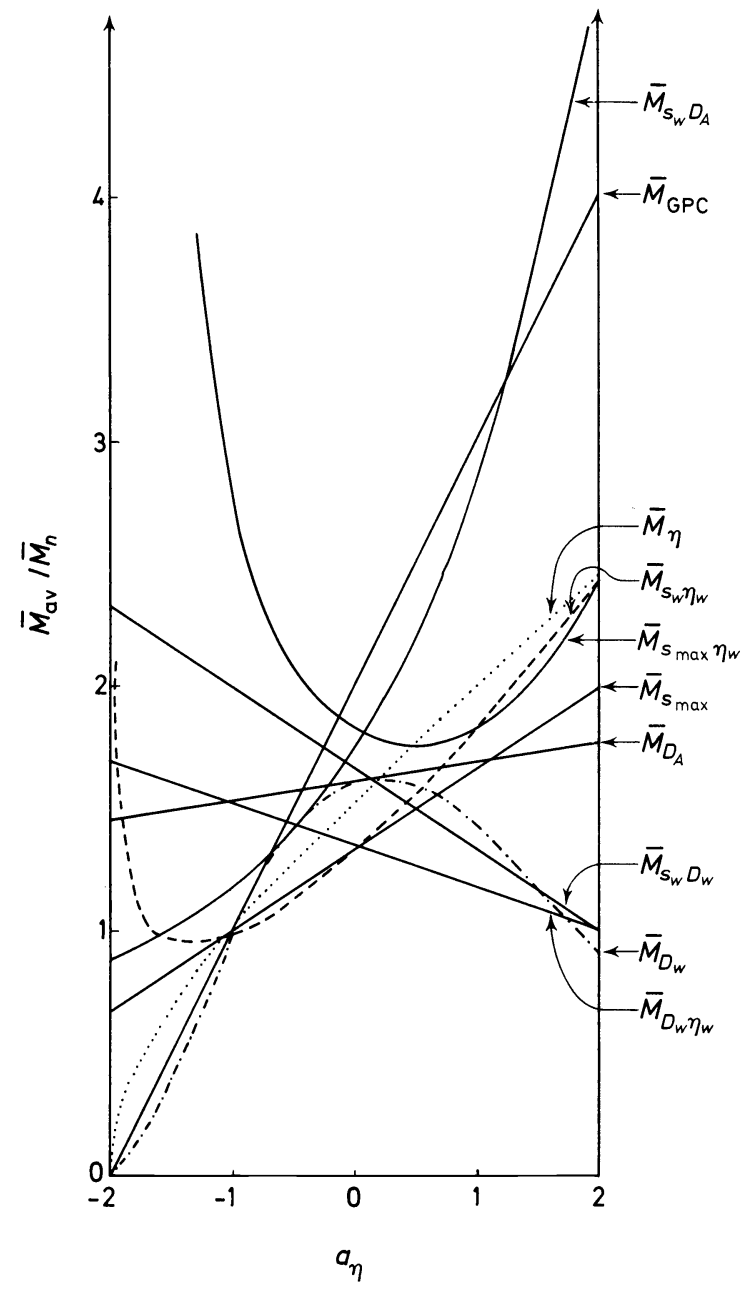

Figure 1. Influence of the parameter $a_{\eta}$ as a measure for the solute shape and the solute/solvent interaction on the numerical values of several mixed average molecular weights for a polymer with a Schulz-Flory molecular weight distribution and a $M_{w} / M_{n}=2($ i.e. $\lambda=1)$ 
average thus depends on the average of the properties (e.g. intrinsic viscosity, sedimentation coefficient, etc.), the type and the width of the molecular weight distribution, and the shape of the solute and its interaction with the solvent. The influence of the latter, e.g. as given by the exponent $a_{\eta}$ of the intrinsic viscosity/molecular weight relationship, can be especially pronounced (Figure 1). Sometimes the mixed averages are identical with simple

Table 3. Averages and moments of molecular weights (molar masses) from Svedberg and Mandelkern-Flory-Scheraga equations for measurements of random coils in the unperturbed state $\left(a_{\eta}={ }_{2}^{1}\right) \cdot D_{A}=\left(\sum_{i} w_{i} D_{i}^{-\frac{1}{2}}\right)^{-2}$ is the so-called area average of the diffusion coefficient. The numerical values refer to a Schulz-Flory 'normal' distribution with $M_{w} / M_{n}=2$ and $M_{n}=100000 \mathrm{~g} \mathrm{~mol}^{-1}$

\begin{tabular}{|c|c|c|}
\hline $\begin{array}{c}\text { Mixed } \\
\text { averages }\end{array}$ & Molecular weight averages or moments & $\begin{array}{l}\text { Numerical value } \\
\quad\left(\mathrm{g} \mathrm{mol}^{-1}\right)\end{array}$ \\
\hline & $M_{n}$ & 100000 \\
\hline$M_{s_{w} D_{w}}^{s_{n} D_{w}}$ & $M_{n}^{n} \mu_{w}^{\left(\frac{1}{2}\right)} / \mu_{n}^{\left(\frac{1}{2}\right)}$ & 150000 \\
\hline$M_{s}^{s_{w}}$ & $M_{\mathrm{w}}$ & 200000 \\
\hline$M_{s_{w} D_{A}}^{s_{w} D_{z}}$ & $\mu_{w}^{\left(\frac{w}{2}\right)}\left(\mu_{w}^{\left(\frac{1}{w}\right)}\right)^{2}$ & 170646 \\
\hline$M_{s_{w} \eta_{w}}^{s_{w} D_{A}}$ & $\left(\mu_{z}^{\left(\frac{1}{2}\right)}\right)^{2^{w}}=M_{\eta}$ & 176716 \\
\hline$M_{D_{w} \eta_{w}}$ & $\left(\mu_{n}^{(1)}\right)^{3} /\left[\left(\mu_{n}^{\left(\frac{1}{2}\right)^{n}}\right)^{3}\left(\mu_{w}^{\left(\frac{1}{2}\right)}\right]\right.$ & 108074 \\
\hline$M_{D} D_{w} \eta_{w}$ & $\left(\mu_{w}^{\left(\frac{1}{2}\right)}\right)^{6} / \mu_{w}^{\left(\frac{1}{2}\right)^{n}}$ & 159126 \\
\hline$M_{\eta}^{D_{A} \eta_{w}}$ & $\left(\mu_{w}^{\left(\frac{1}{2}\right)}\right)^{2}=M_{\eta}$ & 176716 \\
\hline$M_{\mathrm{GPC}}^{\eta}$ & $\left(\mu_{w}^{\left(\frac{2}{2}\right)}\right)\left(\mu_{w}^{\left(\frac{1}{2}\right)}\right)^{-1}$ & 250000 \\
\hline
\end{tabular}

averages. A few examples are shown in Table 3 for the exponent $a_{\eta}$, e.g. for random coils in a theta solvent. Very different molecular weight averages may thus result if the property average is changed (Table 3). Two facts are of special interest. Although many physical equations of those shown in Table 1 clearly give 'absolute molecular weights' (i.e. the molecular weight can be calculated from experimental data without calibration), the resulting mixed averages may depend on the solvent. One may note furthermore that the combination of the weight average of the sedimentation coefficient and the intrinsic viscosity gives directly the viscosity average of the molecular weight for random coils in the unperturbed state ${ }^{69}$. This molecular weight $M_{s_{n} \eta_{w}}$ can thus be used directly to establish intrinsic viscosity/molecular weight relationships without the usual polymolecularity corrections.

Very few experimental data have been reported in literature which can be used to check the predictions made by this simple approach. Molecular weight determinations on a poly(styrene) sample in cyclohexane at $34^{\circ} \mathrm{C}$ (theta conditions) yielded $M_{z}=423000, M_{w}=239000$ and $M_{n}=60000$ $\mathrm{g} \mathrm{mol}^{-1}$, i.e. the distribution can be represented by a Schulz-Flory distribu-

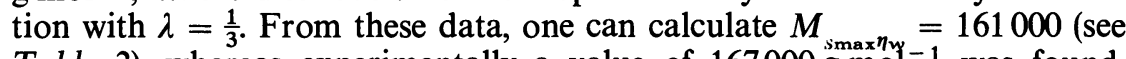
Table 2), whereas experimentally a value of $167000 \mathrm{~g} \mathrm{~mol}^{-1}$ was found. Kotera, Saito and Takemura ${ }^{70}$ determined many mixed averages of molecular weight for a mixture of two samples and compared them with the predicted numerical values. The agreement was good to fair in most cases. It must be mentioned that the unknown molecular weight distribution of the two parent samples may have affected the results to some extent, since the authors assumed the parent samples to be molecular homogeneous. 


\section{Simple averages from combinations of properties}

The previous section was concerned with the problem of which molecular averages result if simple property averages are inserted in one of the physical equations given in Table 1. In many cases, however, one is interested in the opposite problem: how to get a simple molecular weight average from a combination of property averages.

Early papers were mainly concerned with measurements in the theta state (Table 4), although a general solution for the problem of determination of weight average molecular weights from sedimentation coefficient $/ \mathrm{mole}$ cular weight relationships has been known for some time ${ }^{34,71}$.

Table 4. Simple molecular averages from combinations of property averages in complex molecular weight equations for measurements in the theta state. The symbols have the same meaning as in Table 1

\begin{tabular}{clc}
\hline $\begin{array}{c}\text { Molecular weight } \\
\text { average }\end{array}$ & \multicolumn{1}{c}{ Proper combination } & Ref. \\
\hline$M_{n}$ & $K_{s}^{-1}\left(\sum_{i} w_{i} s_{i}^{-2}\right)^{-1}$ & 71,72 \\
$M_{w}$ & $K_{s}^{-1}\left(\sum_{i} w_{i} s_{i}^{2}\right)$ & 73 \\
$M_{w}$ & $f_{s} f_{D}^{-1} R T\left(1-\tilde{v}_{2} \rho_{1}\right)^{-1}\left(\sum_{i} w_{i} s_{i}^{2}\right)\left(\sum_{i} w_{i} s_{i}^{-1}\right)\left(\sum_{i} w_{i} D_{i}\right)^{-1}$ & 74 \\
$M_{w}$ & {$\left[N_{a} \eta \beta\left(1-\tilde{v}_{2} \rho_{1}\right)^{-1}\right]^{\frac{2}{2}}\left(\sum_{i} w_{i} s_{i}^{2}\right)\left(\sum_{i} w_{i} s_{i}^{\frac{2}{3}}\right)^{-\frac{1}{2}}[\eta]^{\frac{1}{2}}$} & 75,76, \\
\hline
\end{tabular}

A general solution for the problem of finding simple averages of molecular weight from combinations of arbitrary averages of other properties has been presented recently ${ }^{77}$ and utilized for the calculation of the weight average molecular weight from sedimentation and diffusion coefficients ${ }^{78}$.

\section{Polymolecularity corrections}

Exact correlations between two different properties can be established only if the proper averages of these properties are used. A well- and longknown example is the correlation between intrinsic viscosity and molecular weight where the intrinsic viscosity is a simple weight average ${ }^{14-16}$ and the molecular weight is the so-called viscosity average ${ }^{16}$, which is an exponential resolved weight average ${ }^{2}$.

Polymolecularity correction factors have to be used if other than the proper property and molecular weight averages are used to establish correlations. These correction factors can range up to several hundred per cent even for distributions that are not very broad, and depend on the type of the property/molecular weight relationship, the type of the molecular weight distribution, the shape of the polymer and the polymer/solvent interaction. 
Polymolecularity corrections are most dramatic for the so-called Baumann equation ${ }^{79}$ :

$$
\begin{aligned}
& \left(\left\langle R^{2}\right\rangle / M\right)^{\frac{3}{2}}=K_{0}^{\frac{3}{2}}+B M^{\frac{1}{2}} \\
& \left\langle R^{2}\right\rangle^{\frac{3}{2}}=K_{0}^{\frac{3}{2}} M^{\frac{3}{2}}+B M^{2}
\end{aligned}
$$

where $\left\langle R^{2}\right\rangle=$ mean square radius of gyration; $K_{0}, B=$ system specific constants. Normally, the radii of gyration are determined as $z$-averages and the molecular weights as weight averages. Polymolecularity correction factors can then be calculated for either the constants $K_{0}$ and $B^{80}$ or (better) for the two variables $\left\langle R_{z}^{2}\right\rangle$ and $M_{w}{ }^{81}$. Taking the $z$-average of equation (40)

$$
\left\langle\left(R^{2}\right)^{\frac{3}{2}}\right\rangle_{z}=\sum_{i} Z_{i}\left(R^{2}\right)_{i}^{\frac{3}{2}}=K_{0}^{\frac{3}{2}} \sum_{i} Z_{i} M_{i}^{\frac{3}{2}}+B \sum_{i} Z_{i} M_{i}^{2}
$$

and comparing this expression with equation (39) written for weight average molecular weight and $z$-average mean square radius of gyration gives the polymolecularity correction factors ${ }^{81}$

$$
\left[\left(\left\langle R_{z}^{2}\right\rangle q_{R_{z}}\right) /\left(M_{w} q_{M_{w}}\right)\right]^{\frac{3}{2}}=K_{0}^{\frac{3}{2}}+B\left(M_{w} q_{M_{w}}\right)^{\frac{1}{2}}
$$

with

$$
\begin{gathered}
q_{R_{z}}=\left(\sum_{i} Z_{i} R_{i}^{3}\right)^{\frac{2}{3}}\left(\sum_{i} Z_{i} M_{i}^{2}\right)^{2} /\left\langle R_{z}^{2}\right\rangle\left(\sum_{i} Z_{i} M_{i}^{\frac{3}{2}}\right)^{\frac{8}{3}} \\
q_{M_{w}}=\left(\sum_{i} Z_{i} M_{i}^{2}\right)^{2} / M_{w}\left(\sum_{i} Z_{i} M_{i}^{\frac{3}{3}}\right)^{2}
\end{gathered}
$$

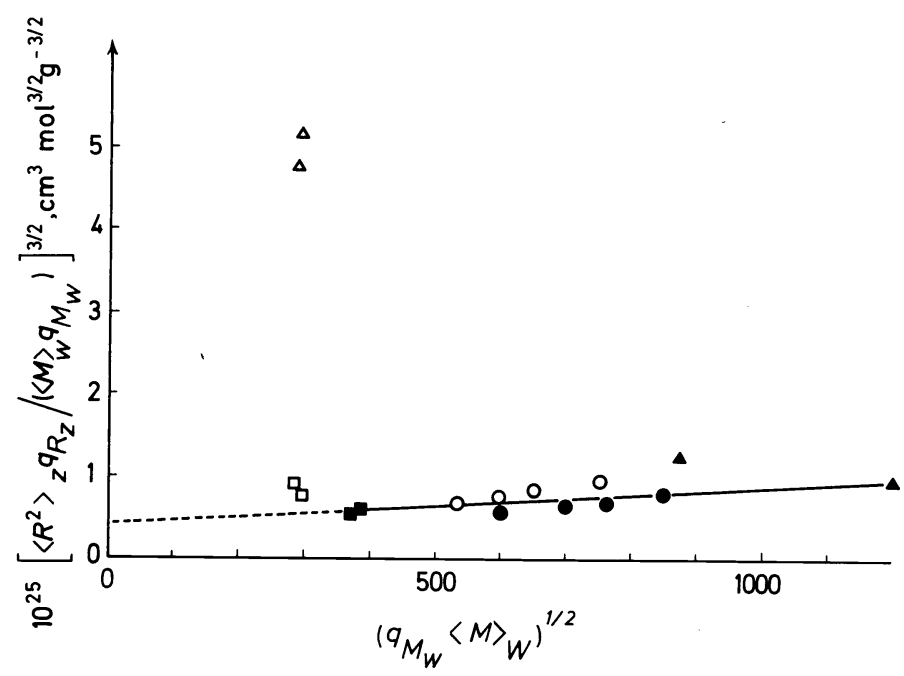

Figure 2. Influence of polymolecularity corrections on the molecular weight dependence of reduced radii of gyration of poly $\left(\alpha\right.$-methylstyrene)s in toluene at $25^{\circ} \mathrm{C}$ (from ref. 83). Uncorrected data $\left(q_{R_{z}}=1 ; q_{M_{w}}=1\right)$ are shown as open symbols, i.e. Schulz-Flory distributions ( $\square$ ), logarithmic normal distributions $(\Delta)$ and 'narrow' distributions $(O)$, whereas data corrected for polymolecularity are given by full symbols, i.e. Schulz-Flory distributions $(\boldsymbol{\square})$, logarithmic normal distributions $(\boldsymbol{\Lambda})$ and 'narrow' distributions ( $(\mathbf{)}$, corrected under assumption of a SchulzFlory distribution 
Equations (43) and (44) contain moments which are not easy to determine experimentally. They can be replaced by experimental quantities if the type of the molecular weight distribution is known.

Figure 2 compares experimental results on poly( $\alpha$-methylstyrene)s in toluene at $25^{\circ} \mathrm{C}$ with and without polymolecularity corrections. The dramatic influence of the polymolecularity corrections on the properties of samples with a logarithmic normal distribution of molecular weights is clearly seen. Many papers in literature contain remarks on polymolecularity correction factors; most of them are improper. Correct polymolecularity correction factors were given for the Kuhn-Mark-Houwink-Sakurada equation

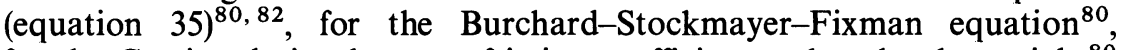
for the Cowie relation between friction coefficient and molecular weight ${ }^{80}$ and for the above-mentioned Baumann equation ${ }^{80,81,83}$. Polymolecularity correction factors were reviewed ${ }^{2}$ and tabulated ${ }^{84}$ in detail.

\section{VIRIAL COEFFICIENT AVERAGES}

The definitions of the second virial coefficient averages, equations (2) and (3), predict an $\left(A_{2}\right)^{\mathrm{OP}} \geqslant\left(A_{2}\right)^{\mathrm{LS}}$ which has been verified experimentally ${ }^{85-88}$. In the case of mixtures of two molecular homogeneous polymers, equations (2) and (3) can be reduced to

$$
\begin{aligned}
& \left(A_{2}\right)^{\mathrm{OP}}=\left(A_{2}\right)_{i i} w_{i}^{2}+2\left(A_{2}\right)_{i j} w_{i} w_{j}+\left(A_{2}\right)_{j j} w_{j}^{2} \\
& \left(A_{2}\right)^{\mathrm{LS}}=\left[\left(A_{2}\right)_{i i} M_{i}^{2} w_{i}^{2}+2\left(A_{2}\right)_{i j} M_{i} M_{j} w_{i} w_{j}+\left(A_{2}\right)_{j j} M_{j}^{2} w_{j}^{2}\right] / M_{w}^{2}
\end{aligned}
$$

where $\left(A_{2}\right)_{i i}$ and $\left(A_{2}\right)_{j j}$ are the two second virial coefficients for the molecular homogeneous polymers $i$ and $j, w_{i}$ and $w_{j}$ the corresponding mass fractions and $\left(A_{2}\right)_{i j}$ the so-called cross virial coefficient.

Equations (45) and (46) predict maxima in the functions $A_{2}^{\mathrm{OP}}=f\left(w_{j}\right)$ and $A_{2}^{\mathrm{LS}}=f\left(w_{j}\right)$, if $\left(A_{2}\right)_{i i}>\left(A_{2}\right)_{i j}$ or $\left(A_{2}\right)_{i i}>\left(A_{2}\right)_{j j}$ (see reference 89). The second virial coefficients decrease with increasing molecular weight in a homologous polymer series according to

$$
A_{2}=K_{\mathrm{A}} M^{-a_{A}}
$$

The predicted maxima have been found by some authors ${ }^{90-92}$, but not by others ${ }^{93,94}$. Several reasons might be responsible for this discrepancy: (a) the polymers $i$ and $j$ were not homogeneous and the averaging might thus have been improper; (b) the evaluation of the second virial coefficients was incorrect, e.g. by using a certain relationship between the second and the third virial coefficient; and (c) the maximum may lie at very low values of $w_{j}$ and thus be difficult to detect.

The effect of the type and width of the molecular weight distribution was investigated only recently ${ }^{95}$. Many samples of poly( $\alpha$-methylstyrene) were mixed together to obtain a preselected distribution function, and with the weight average molecular weight held constant $\left(M_{w}=80000 \mathrm{~g} \mathrm{~mol}^{-1}\right)$. The second virial coefficients from light scattering measurements were about constant and independent of the varying number average molecular weight and the type of the distribution. With increasing polymolecularity parameters, the osmotic second virial coefficients of the mixtures with 


\section{HANS-GEORG ELIAS}

Schulz-Flory distributions decreased, whereas the corresponding osmotic second virial coefficients of mixtures with logarithmic normal distributions increased much more rapidly than predicted by the hard sphere approximation theory (Figure 3). Replacement of the hard sphere approximations by a soft sphere model $^{96}$, i.e. a model with overlapping random coils, does not lessen the disagreement between experimental and theoretical second virial coefficients. The observed discrepancy might well be due to a breakdown of equation (47) for low molecular weights. Further theoretical and experimental work is clearly needed in this area.

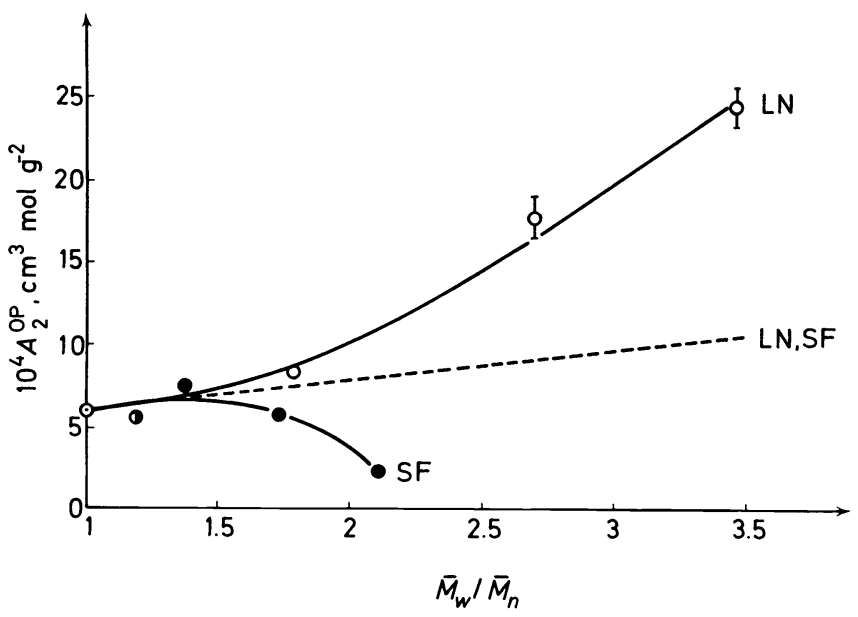

Figure 3. Dependence of osmotic second virial coefficient $A_{L}^{\mathrm{OP}}$ on the polymolecularity index $\bar{M}_{w} / \bar{M}_{n}$ for poly $\left(\alpha\right.$-methylstyrene)s in toluene at $25^{\circ} \mathrm{C}$ with constant weight average molecular weight $M_{w} \approx 80000 \mathrm{~g} \mathrm{~mol}^{-1}$. The symbols indicate samples with logarithmic normal distribution of molecular weights $(\bigcirc)$, Schulz-Flory distributions $(\bullet)$, a narrow distributed sample with unknown type of distribution function ( $(\boldsymbol{\Phi})$ and the data point from the calibration curve for a hypothetical molecular homogeneous sample $(\odot)$. The dotted line (----) indicates the prediction of the hard sphere approximation. (From ref. 95)

\section{INFLUENCE OF POLYMOLECULARITY ON ASSOCIATION}

\section{Distinction between types of association}

Association equilibria can be conveniently described by two limiting cases and combinations thereof: open and closed association ${ }^{89}$. Open association is defined as an association of unimers with molecular weight $M_{\mathrm{I}}$ to an unlimited number of multimers:

$$
\begin{aligned}
& 2 M_{\mathrm{I}} \rightleftarrows M_{\mathrm{II}} \\
& M_{\mathrm{II}}+M_{\mathrm{I}} \rightleftarrows M_{\mathrm{III}} \\
& M_{\mathrm{III}}+M_{\mathrm{I}} \rightleftarrows M_{\mathrm{IV}}, \text { etc. }
\end{aligned}
$$


Closed association, on the other hand, is characterized by an equilibrium between two distinct species only, unimer and $r$-mer:

$$
r M_{\mathrm{I}} \rightleftarrows M_{r}
$$

Both of these limiting cases can be distinguished easily by their characteristic concentration dependence of apparent molecular weights in the low concentration range (Figure 4). Only closed associations show a characteristic 'critical micelle concentration' (cmc). Distinctions between closed and open associations are less easily made if the whole concentration dependence of the apparent molecular weights is unknown, especially at low concentrations. In this case, both the concentration dependencies of the inverse apparent molecular weight for open and closed associations look like hyperbolic curves (Figure 4).

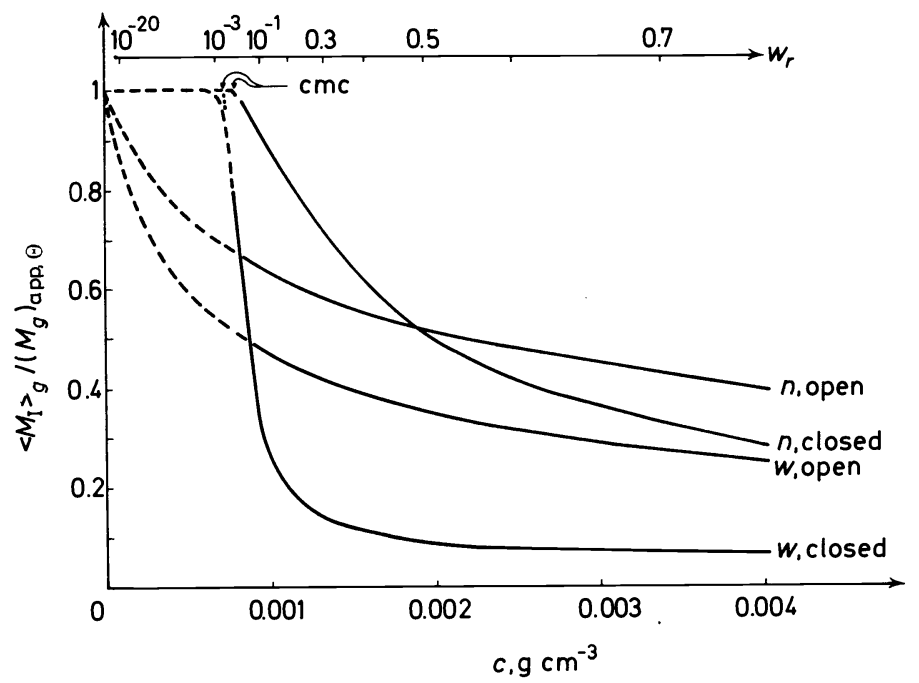

Figure 4. Concentration dependence of the normalized inverse apparent $g$-average molecular weight in the theta state for molecule-based closed associations $\left(r=21 ;{ }^{n} K_{c}=10^{45}\left(\mathrm{dm}^{3}\right.\right.$ $\left.\left.\mathrm{mol}^{-1}\right)^{20} ;\left(M_{\mathrm{I}}\right)_{n}=\left(M_{\mathrm{I}}\right)_{w}=\left(M_{\mathrm{I}}\right)_{z}=200 \mathrm{~g} \mathrm{~mol}^{-1}\right)$ and the equivalent open association with ${ }^{n} K_{0}=\left({ }^{n} K_{c}\right)^{1 /(r-1)} \cdot w_{r}=c_{r} / c=$ mass fraction of the $r$-mer. Solid lines correspond to (hypothetically) experimentally accessible data, broken lines to data predicted by the model. The 'critical micelle concentrations' cmc depend on the method and do not correspond to a 'first appearance' of micelles. (Adapted from ref. 97)

Open and closed molecule-based associations (see below) can be distinguished from each other, however, if, e.g., the apparent weight average molecular weight is plotted against the inverse number average molecular weight $^{97}$ (see Figure 5). For closed associations, straight lines are observed for theta systems, since

$$
\left(M_{w}\right)_{\text {app }, \theta}=\left\langle M_{\mathrm{I}}\right\rangle_{w}+r\left\langle M_{\mathrm{I}}\right\rangle_{n}-r\left\langle M_{\mathrm{I}}\right\rangle_{n}^{2}\left(M_{n}\right)_{\mathrm{app}, \theta}^{-1}
$$




\section{HANS-GEORG ELIAS}

Concave lines are observed for closed associations in non-theta systems. On the other hand, this plot always gives convex lines for open associations in both theta and non-theta systems, as shown by the following equation for open associations of rods under theta conditions (see reference 98):

$$
\left(M_{w}\right)_{\text {app }, \theta}=\left\langle M_{1}\right\rangle_{w}-2\left\langle M_{1}\right\rangle_{n}+2\left(M_{n}\right)_{\text {app }, \theta}
$$

Similar relationships can be derived for apparent $z$-averages. For closed molecule-based associations, one obtains

$$
\begin{aligned}
\left(M_{z}\right)_{\mathrm{app}, \theta}=\left\langle M_{\mathrm{I}}\right\rangle_{z}+\left[1+2\left\langle M_{\mathrm{I}}\right\rangle_{w}-\left\langle M_{\mathrm{I}}\right\rangle_{n}-\left\langle M_{\mathrm{I}}\right\rangle_{z}\right] & \\
& {\left[1-\left\langle M_{\mathrm{I}}\right\rangle_{w}\left(M_{w}\right)_{\mathrm{app}, \theta}^{-1}\right] }
\end{aligned}
$$

and for open molecule-based associations

$$
\left(M_{z}\right)_{\text {app }, \theta}\left(M_{w}\right)_{a p p, \theta}=\left\langle M_{\mathrm{I}}\right\rangle_{w}\left[\left\langle M_{\mathrm{I}}\right\rangle_{z}-\left(\frac{3}{2}\right)\left\langle M_{\mathrm{I}}\right\rangle_{w}\right]+\left(\frac{3}{2}\right)\left(M_{w}\right)_{\mathrm{app}, \theta}^{2}
$$

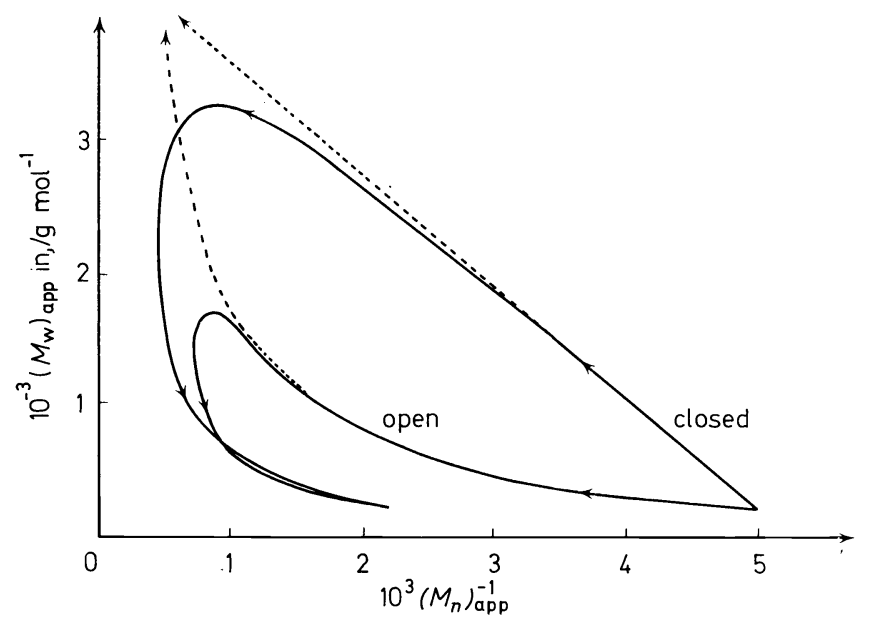

Figure 5. Apparent weight average molecular weights in the theta state as a function of the inverse apparent number average molecular weight in the theta state for closed and open associations with the same molecule-based open association equilibrium constant ${ }^{n} K_{0}$. Data of Figure 4: ${ }^{n} K_{0}=\left({ }^{n} K_{c}\right)^{1 /(r-1)} ;{ }^{n} K_{c}=10^{45}\left(\mathrm{dm}^{3} \mathrm{~mol}^{-1}\right)^{20} ; r=21 ;\left(M_{\mathrm{I}}\right)_{n}=\left(M_{\mathrm{I}}\right)_{w}=\left(M_{\mathrm{I}}\right)_{z}=200$ $\mathrm{g} \mathrm{mol}^{-1}$. Broken lines correspond to theta state conditions, solid lines to end-to-end association of rigid cylindrical rods with a radius of $5 \times 10^{-8} \mathrm{~cm}$ and a specific volume of $1 \mathrm{~cm}^{3} \mathrm{~g}^{-1}$. The concentration increases in the direction of the arrows. (From ref. 97)

Both open and closed associations can be either molecule- or segmentbased.

A molecule-based association is defined as an association of molecules whose number of associogenic sites is independent of molecular weight. A common example is association via the two end-groups of tinear molecules. It is convenient and in many cases justified by either experiment or chemical intuition, to assume the equilibrium constants to be independent of the particle size (principle of equal physical reactivities). This assumption 
allows the treatment of open associations with only a single equilibrium constant.

A segment-based association is defined as an association of molecules whose number of associogenic sites increase linearly with the molecular weight. An example might be the association of partially stereoregular molecules whose number of stereoregular segments increase with molecular weight.

Molecule- and segment-based open associations can be distinguished in certain cases, particularly if statistical weights of higher rank are used (see reference 98). The distinction is possible because the relation

$$
\left({ }^{w} K_{0}\right)=r(r-1)^{-1}\left\langle M_{\mathrm{I}}\right\rangle_{n}^{-1}\left({ }^{n} K_{0}\right)=Q\left\langle M_{\mathrm{I}}\right\rangle_{n}^{-1}\left({ }^{n} K_{0}\right)
$$

varies with the average numerical values of $r$, which in turn varies with concentration. The factor $Q$ is equal to 2 for a unimer/dimer association and approaches 1 for the limit of infinite $r .{ }^{w} K_{0}$ and ${ }^{n} K_{0}$ are the equilibrium constants of open association on a mass or mole basis, respectively, where $c=$ mass concentrations and $[M]=$ mole concentrations:

$$
\begin{gathered}
{ }^{w} K_{0} \equiv c_{r} /\left(c_{r-1} c_{\mathrm{I}}\right) \\
{ }^{n} K_{0} \equiv\left[M_{r}\right] /\left(\left[M_{r-1}\right]\left[M_{\mathrm{I}}\right]\right)
\end{gathered}
$$

Molecule- and segment-based closed associations cannot be distinguished for a given sample since there is only one $r$, which, by definition, stays constant over the whole concentration range. If, however, a molecular homologous series is available (i.e. a series of compounds with the same physical reactivity but different polymolecularities), then a distinction can be made on the basis of their polymolecularity/polydispersity relationships (see below).

\section{Influence of polymolecularity on polydispersity}

An $r$-mer can be formed by any $r$ unimer molecules of a polymolecular unimeric compound. The mathematical treatment is especially simple if the physical reactivity is independent of the molecular weight. The relationships between the $g$-averages of the molecular weights of unimers and multimers obviously depend on type of association, i.e. whether it is a molecule-based or a segment-based association.

For molecule-based associations, the number average of the $r$-mer is just $r$ times the number average molecular weight of the unimer:

$$
\left\langle M_{r}\right\rangle_{n}=r\left\langle M_{\mathrm{I}}\right\rangle_{n}
$$

The weight average molecular weight of the $r$-mer is, however, not a simple multiple of the weight average molecular weight of the unimer ${ }^{98}$

$$
\left\langle M_{r}\right\rangle_{w}=\left\langle M_{\mathrm{I}}\right\rangle_{w}+(r-1)\left\langle M_{\mathrm{I}}\right\rangle_{n}
$$

and similarly for the $z$-average ${ }^{98}$

$$
\begin{aligned}
\left\langle M_{r}\right\rangle_{z}=\left\langle M_{\mathrm{I}}\right\rangle_{z}+(r-1)\left\langle M_{\mathrm{I}}\right\rangle_{n} & \left\{1+\left[2\left\langle M_{\mathrm{I}}\right\rangle_{w}-\left\langle M_{\mathrm{I}}\right\rangle_{n}\right.\right. \\
& \left.\left.-\left\langle M_{\mathrm{I}}\right\rangle_{z}\right]\left[\left\langle M_{\mathrm{I}}\right\rangle_{w}+(r-1)\left\langle M_{\mathrm{I}}\right\rangle_{n}\right]^{-1}\right\}
\end{aligned}
$$




\section{HANS-GEORG ELIAS}

For segment-based associations, general expressions have been derived for the weight and the $z$-average of particle weight of the $r$-mers ${ }^{98}$, whereas for the number average a closed expression exists for the case of SchulzFlory distributions only ${ }^{98}$ :

$$
\begin{aligned}
& \left\langle M_{r}\right\rangle_{n}=\left\langle M_{\mathrm{I}}\right\rangle_{n}+(r-1)\left\langle M_{\mathrm{I}}\right\rangle_{w} \quad \text { (Schulz-Flory only) } \\
& \left\langle M_{r}\right\rangle_{w}=r\left\langle M_{\mathrm{I}}\right\rangle_{w} \\
& \left\langle M_{r}\right\rangle_{z}=\left\langle M_{\mathrm{I}}\right\rangle_{z}+(r-1)\left\langle M_{\mathrm{I}}\right\rangle_{w}
\end{aligned}
$$

From equations $(55)-(60)$, the following equations can be derived for the relations between the polymolecularity parameter $\left\langle M_{\mathrm{I}}\right\rangle_{w} /\left\langle M_{\mathrm{I}}\right\rangle_{n}$ and the polydispersity parameter $\left\langle M_{r}\right\rangle_{w} /\left\langle M_{r}\right\rangle_{n}$.

For molecule-based associations:

$$
\left\langle M_{r}\right\rangle_{w} /\left\langle M_{r}\right\rangle_{n}=1+r^{-1}\left[\left\langle M_{\mathrm{I}}\right\rangle_{w} /\left\langle M_{\mathrm{I}}\right\rangle_{n}-1\right]
$$

For segment-based associations with a Schulz-Flory distribution of unimers:

$$
\left\langle M_{r}\right\rangle_{n} /\left\langle M_{r}\right\rangle_{w}=1+r^{-1}\left[\left\langle M_{\mathrm{I}}\right\rangle_{n} /\left\langle M_{\mathrm{I}}\right\rangle_{w}-1\right]
$$

or

$$
\left\langle M_{r}\right\rangle_{w} /\left\langle M_{r}\right\rangle_{n}=\left[\left\langle M_{\mathrm{I}}\right\rangle_{w} /\left\langle M_{\mathrm{I}}\right\rangle_{n}\right] /\left[1-(1-r)\left(\left\langle M_{\mathrm{I}}\right\rangle_{w} /\left\langle M_{\mathrm{I}}\right\rangle_{n}\right)\right]
$$

Diagrams of these polydispersity/polymolecularity relationships (Figures 6 and 7) show that association is accompanied by a pronounced sharpening of the distributions. This effect has the same origin as the one observed for the growing of many chains from one common growth centre ${ }^{99}$ : the distributions are transferred into one particle and the distributions over all particles are thus more homogeneous. The effect is of course the more pronounced the higher the association number $r$. Segment-based associations always

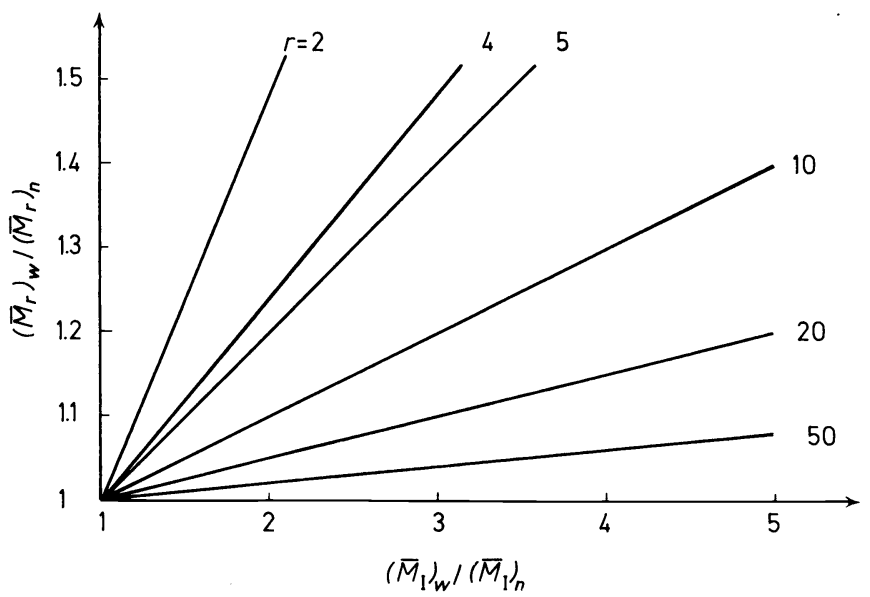

Figure 6. Influence of polymolecularity index $\left(M_{1}\right)_{w} /\left(M_{1}\right)_{n}$ on the polydispersity index $\left(M_{r}\right)_{w} /\left(M_{r}\right)_{n}$ for molecule-based associations for various association numbers $r$. The relationships are independent of the particular type of molecular weight distribution. (From ref. 12) 


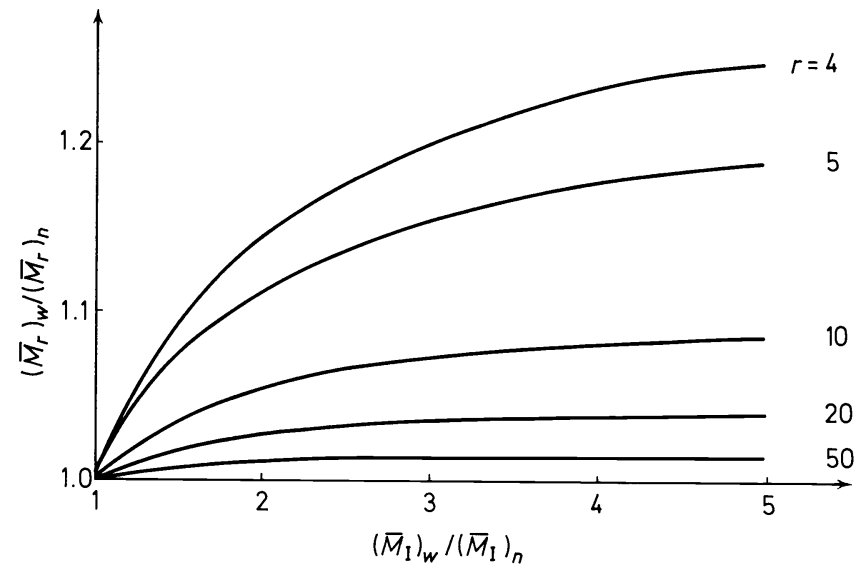

Figure 7. Influence of polymolecularity index $\left(M_{\mathrm{I}}\right)_{w} /\left(M_{\mathrm{I}}\right)_{n}$ on the polydispersity index $\left(M_{r}\right)_{w} /\left(M_{r}\right)_{n}$ for segment-based associations for various association numbers $r$. The relationships are independent of the particular type of molecular weight distribution. (From ref. 12)

show more sharpening than molecule-based associations (Table 5). The polydispersity parameter increases with the polymolecularity parameter for both molecule- and segment-based associations. In the latter case, but not in the former, an asymptote of $\left\langle M_{r}\right\rangle_{w} /\left\langle M_{r}\right\rangle_{n}=r /(r-1)$ is reached for an infinitely high polymolecularity parameter.

Table 5. Influence of polymolecularity parameter $\left\langle M_{\mathrm{I}}\right\rangle_{w} /\left\langle M_{\mathrm{I}}\right\rangle_{n}$ and association number $r$ on the polydisperity parameter $\left\langle M_{r}\right\rangle_{w} /\left\langle M_{r}\right\rangle_{n}$

\begin{tabular}{crcc}
\hline & & \multicolumn{2}{c}{$\left\langle M_{r}\right\rangle_{w} /\left\langle M_{r}\right\rangle_{n}$} \\
$\left\langle M_{\mathrm{I}}\right\rangle_{w} /\left\langle M_{\mathrm{I}}\right\rangle_{n}$ & $r$ & Molecule-based & Segment-based \\
\hline 1.1 & 2 & 1.050 & 1.048 \\
1.1 & 10 & 1.010 & 1.009 \\
1.1 & 100 & 1.001 & 1.001 \\
2 & 2 & 1.500 & 1.333 \\
2 & 10 & 1.100 & 1.053 \\
2 & 100 & 1.010 & 1.005 \\
10 & 2 & 5.500 & 1.818 \\
10 & 10 & 1.900 & 1.099 \\
10 & 100 & 1.090 & 1.009 \\
\hline
\end{tabular}

\section{POLYDISPERSITY OF ASSOCIATED SOLUTES}

\section{Detection of polydispersity in open associations}

Open associations automatically lead to a polydispersity of the $r$-mers, whether the unimer is molecularly homogeneous or polymolecular. It 
remains to show that the polydispersity of the associate corresponds to the one predicted for an open molecule-based association with equal physical reactivity of the unimers and $r$-mers.

In this particular case, one obtains for the concentration dependence of the different apparent molecular weight averages in the theta state ${ }^{98}$ :

$$
\begin{aligned}
& {\left[\left(M_{n}\right)_{\mathrm{app}, \theta}\right]=\left\langle M_{\mathrm{I}}\right\rangle_{n}+\left({ }^{n} K_{0}\right)\left\langle M_{\mathrm{I}}\right\rangle_{n}\left[c\left(M_{n}\right)_{\mathrm{app}, \theta}^{-1}\right]} \\
& {\left[\left(M_{w}\right)_{\mathrm{app}, \theta}\right]=\left\langle M_{\mathrm{I}}\right\rangle_{w}+2\left({ }^{n} K_{0}\right)\left\langle M_{\mathrm{I}}\right\rangle_{n}\left[c\left(M_{n}\right)_{\mathrm{app}, \theta}^{-1}\right]} \\
& {\left[\left(M_{z}\right)_{\mathrm{app}, \theta}\right]=\left(\left\langle M_{\mathrm{I}}\right\rangle_{z}+3\left({ }^{n} K_{0}\right)\left\langle M_{\mathrm{I}}\right\rangle_{n}\left[c\left(M_{n}\right)_{\mathrm{app}, \theta}^{-1}\right]\right)\left\langle M_{\mathrm{I}}\right\rangle_{w}\left[\left(M_{w}\right)_{\mathrm{app}, \theta}^{-1}\right]} \\
& +3\left({ }^{n} K_{0}\right)\left\langle M_{\mathrm{I}}\right\rangle_{n}\left[c\left(M_{n}\right)_{\mathrm{app}, \theta}^{-1}\right]
\end{aligned}
$$

Introducing the apparent association numbers (with $g=n, w, z$, etc.)

$$
\left(Z_{g}\right)_{\mathrm{app}, \theta} \equiv\left(M_{g}\right)_{\mathrm{app}, \theta} /\left\langle M_{\mathrm{I}}\right\rangle_{g}
$$

one arrives at

$$
\left[\left(Z_{w}\right)_{\text {app }, \theta}\right]=1+2\left(\left\langle M_{\mathrm{I}}\right\rangle_{w} /\left\langle M_{\mathrm{I}}\right\rangle_{n}\right)\left[\left(Z_{n}\right)_{\mathrm{app}, \theta}-1\right]
$$

and

$$
\left[\left(Z_{z}\right)_{\mathrm{app}, \theta}\left(Z_{w}\right)_{\mathrm{app}, \theta}\right]=1+\left(\frac{3}{2}\right)\left(\left\langle M_{\mathrm{I}}\right\rangle_{w} /\left\langle M_{\mathrm{I}}\right\rangle_{z}\right)\left[\left(Z_{w}\right)_{\mathrm{app}, \theta}^{2}-1\right]
$$

By plotting the quantities in brackets against each other, a straight line should be observed, if the data can be described by the model of an open molecule-based association with equal physical reactivity of the species. Equation (68) resembles (but does not equal) the relation between weight and number average degrees of polymerization for condensation polymerizations (see, e.g., reference 100), i.e. for the so-called most probable distributions (a special type of a Schulz-Flory distribution)

$$
\langle X\rangle_{w}=1+2\left(\langle X\rangle_{n}-1\right)
$$

Comparison of equations (68) and (70) shows that the presence of a polymolecularity of the solute, expressed by $\left\langle M_{\mathrm{I}}\right\rangle_{w} /\left\langle M_{\mathrm{I}}\right\rangle_{n}$, increases the apparent polydispersity of associating solutes relative to the polymolecularity of polycondensates. In the limiting case of molecular homogeneous unimers or monomers, both equations become identical.

For segment-based associations, the concentration dependencies of the apparent weight and $z$-averages of molecular weight in the theta state can be given with complete generality ${ }^{98}$ :

$$
\begin{aligned}
& \left(M_{z}\right)_{\mathrm{app}, \theta}=\left\langle M_{\mathrm{I}}\right\rangle_{z}+2\left({ }^{w} K_{0}\right)\left(M_{\mathrm{I}}\right)_{w} c \\
& \left(M_{w}\right)_{\mathrm{app}, \theta}=\left\langle M_{\mathrm{I}}\right\rangle_{w}+\left({ }^{w} K_{0}\right)\left(M_{\mathrm{I}}\right)_{w} c
\end{aligned}
$$

whereas the concentration dependence of the apparent number average molecular weights can be described by a simple equation only for the case of a molecular homogeneous unimer:

$$
\left(M_{n}\right)_{\mathrm{app}, \theta}=\left\langle M_{\mathrm{I}}\right\rangle_{n}\left({ }^{w} K_{0}\right) c\left[\ln \left(1+\left({ }^{w} K_{0}\right) c\right]^{-1}\right.
$$

The combination of equations (71) and (72) leads to

$$
\left(Z_{z}\right)_{\mathrm{app}, \theta}=1+2\left[\left(Z_{w}\right)_{\mathrm{app}, \theta}-1\right]
$$




\section{Detection of polydispersity in closed associations}

The simplest model for a closed association assumes that only unimer and $r$-mers coexist in equilibrium. Because the relative concentration of these two species varies with the total concentration, weight average methods will invariably give higher apparent molecular weights than number average methods in the concentration range between the "critical micelle concentration' and the usually employed highest concentrations (see also Figure 4). A difference in different apparent molecular weights can thus a priori not be interpreted as a polydispersity of the associates.

At very high concentrations, all apparent molecular weight averages become identical for measurements in the theta state, if the simple unimer/ $r$-mer equilibrium applies for molecularly homogeneous unimers. The situation again becomes complicated if the second and higher virial terms cannot be neglected.

For polymolecular unimers undergoing a simple unimer/ $r$-mer association in the theta state, the ratio $\left(M_{w}\right)_{\text {app, } \theta} /\left(M_{n}\right)_{\text {app, } \theta}$ will equal the polydispersity parameter $\left\langle M_{r}\right\rangle_{w} /\left\langle M_{r}\right\rangle_{n}$ at very high concentrations. As may be seen from Table 5 , the polydispersity parameter is always smaller than the polymolecularity parameter of the unimer and very often indistinguishable from 1 within the limits of error of the experiment. It is thus doubtful whether any information on polydispersity or polymolecularity can be drawn from this type of experiment, particularly if the whole concentration dependence of apparent molecular weights is unknown and/or virial coefficients have to be considered.

\section{Multiple equilibria in closed associations}

The previous discussion was concerned with simple closed associations, i.e. associations between a unimer and a single $r$-mer. Combinations of different closed associations or of a closed and an open association may occur, of course. A very simple and often-suggested case is the so-called premicellar equilibrium. This name is given to a combination of two consecutive equilibria, e.g.

$$
\begin{gathered}
q M_{\mathrm{I}} \rightleftarrows M_{q} ;{ }^{n} K_{q}=\left[M_{q}\right] /\left[M_{\mathrm{I}}\right]^{q} \\
r / q M_{q} \rightleftarrows M_{r} ;{ }^{n} K_{r / q}=\left[M_{r}\right] /\left[M_{q}\right]^{r / q}
\end{gathered}
$$

$q$ is generally considered to be small, e.g. 2 or 3 , whereas $r$ is high, typically between 10 and 100. Any other definition can be used, too, e.g.

$$
\begin{aligned}
& q M_{\mathrm{I}} \rightleftarrows M_{q} ;{ }^{n} K_{q}=\left[M_{q}\right] /\left[M_{\mathrm{I}}\right]^{q} \\
& r M_{\mathrm{I}} \rightleftarrows M_{r} ;{ }^{n} K_{r}=\left[M_{r}\right] /\left[M_{\mathrm{I}}\right]^{r}
\end{aligned}
$$

All definitions lead to

$$
{ }^{n} K_{c} \equiv{ }^{n} K_{r} \equiv\left[M_{r}\right] /\left[M_{1}\right]^{r}=\left({ }^{n} K_{r / q}\right)\left({ }^{n} K_{q}\right)^{r / q}
$$

Equations (75), (76) or (77), (78) should in principle lead to two-step curves in the $\left(M_{g}\right)_{\mathrm{app}, \theta}=f(c)$ diagrams. Whether these two-step curves can be observed experimentally will of course depend on the relative magnitude of the two association numbers $q$ and $r$ and the two equilibrium constants 


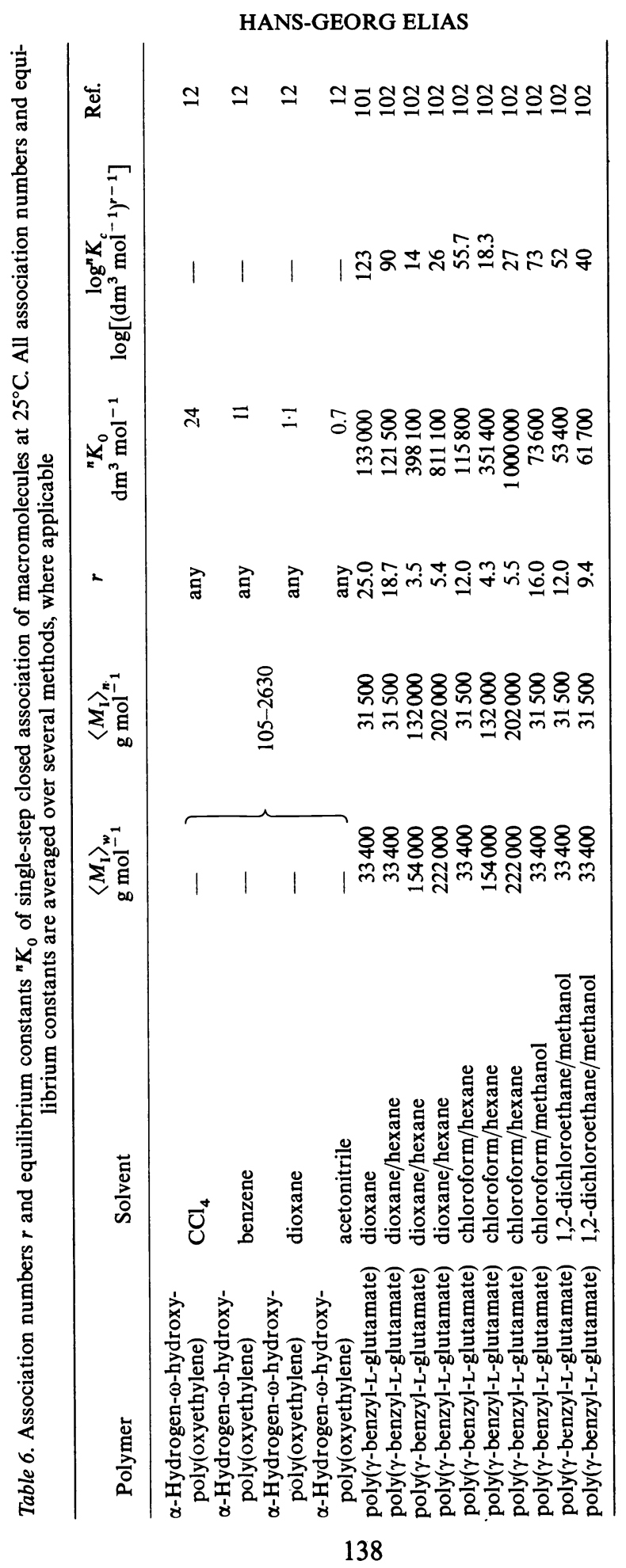




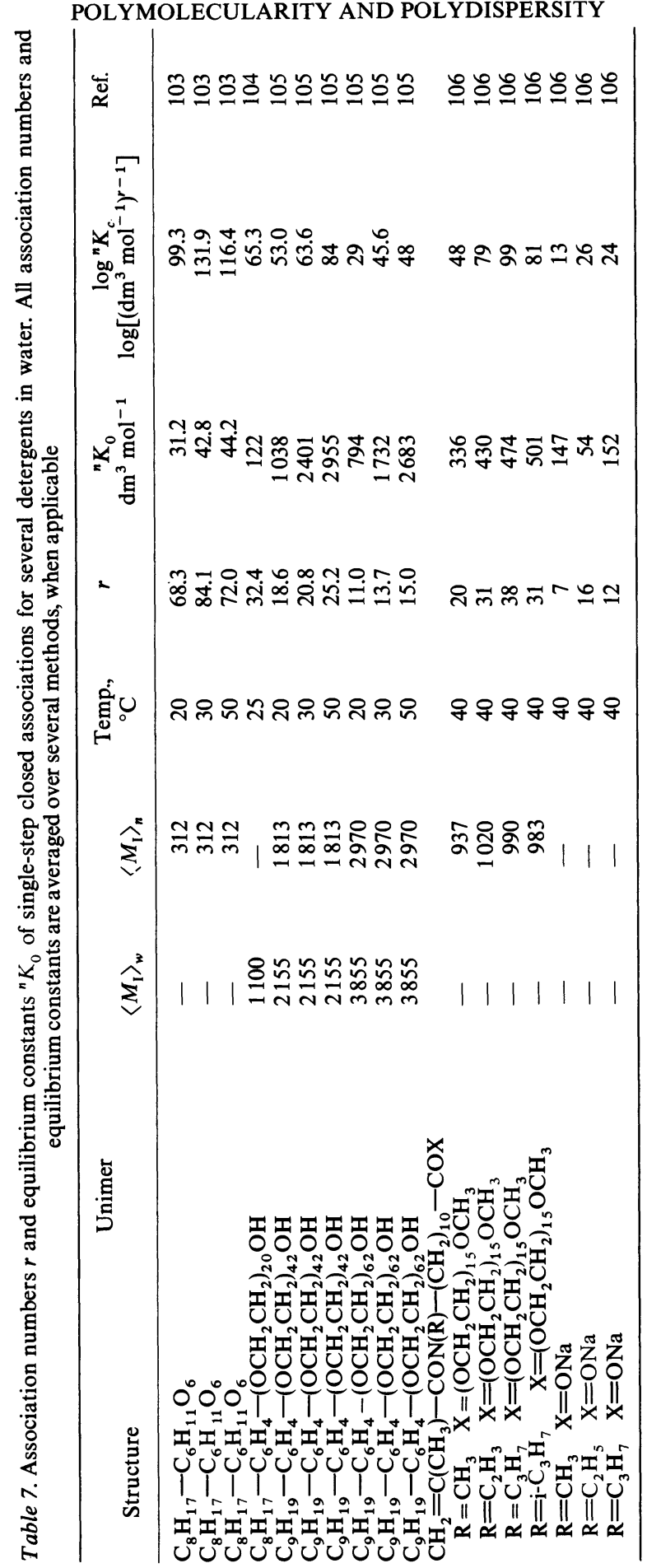




\section{HANS-GEORG ELIAS}

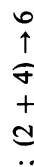

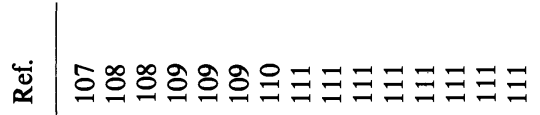

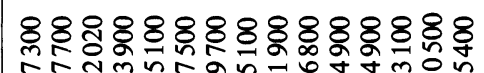

其

马ु

ฮี

is

을

.2

음

.ัँّ

总

प.

\%

도

เุ

을

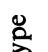

हुं

NNNNNNNNNNNNNNN

$\uparrow \uparrow \uparrow \uparrow \uparrow \uparrow \uparrow \uparrow \uparrow \uparrow \uparrow \uparrow \uparrow \uparrow \uparrow$

足

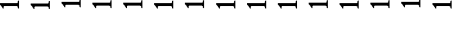

$\Xi$

:

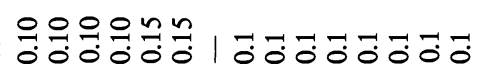

.

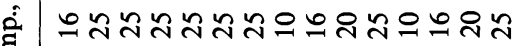

惫

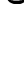

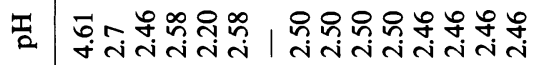

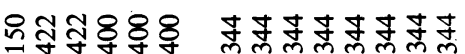

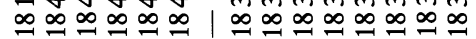




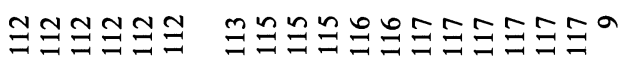

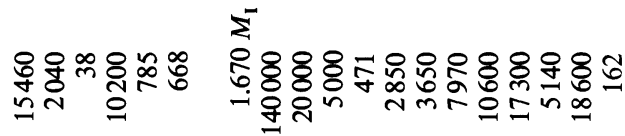

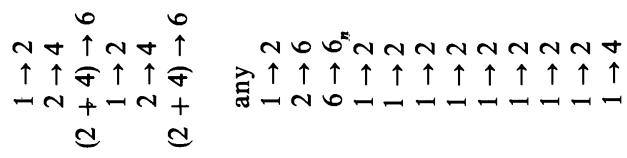

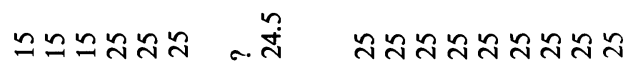

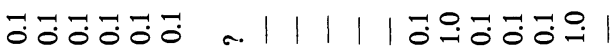

엉유유유

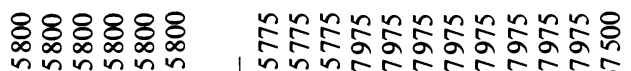

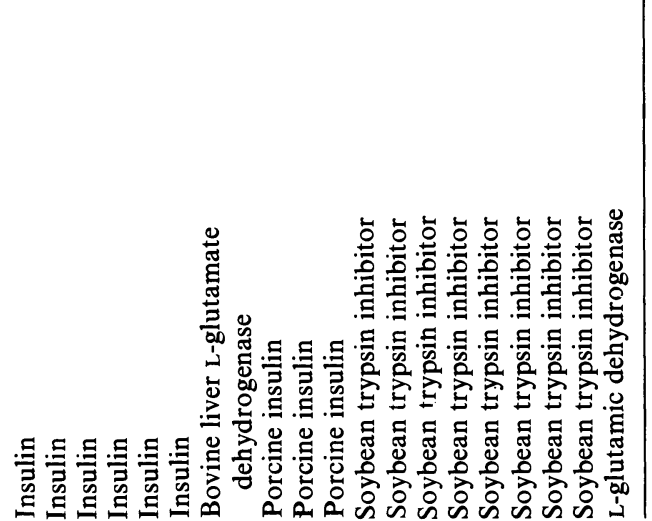


${ }^{n} K_{q}$ and ${ }^{n} K_{r / q}$. Model calculations should thus be based on 'reasonable' values, i.e. those resembling data from experiments. It is noteworthy that only very few equilibrium constants of association have been reported in literature. Those for polymers are collected in Table 6, those for detergents in Table 7 and those for proteins in Table 8. Based on these tables, equilibrium constants of ${ }^{n} K_{q}=2500 \mathrm{dm}^{3} \mathrm{~mol}^{-1}$ and ${ }^{n} K_{0}=\left({ }^{n} K_{r / q}\right)^{1 /\left(r q^{-1}-1\right)} \approx 3464$ $\mathrm{dm}^{3} \mathrm{~mol}^{-1}$ with ${ }^{q} K_{r / q}=50^{50}\left(\mathrm{dm}^{3} \mathrm{~mol}^{-1}\right)^{24}$ and association numbers of $q=2$ and $r=50$ seem to be reasonable for model calculations. The normalized $\left(M_{g}\right)_{\text {app, } \theta}^{-1}=f(c)$ curves do indeed show the two-step character, the transition at the critical micelle concentration being less pronounced as compared with a one-step closed association (Figure 8).

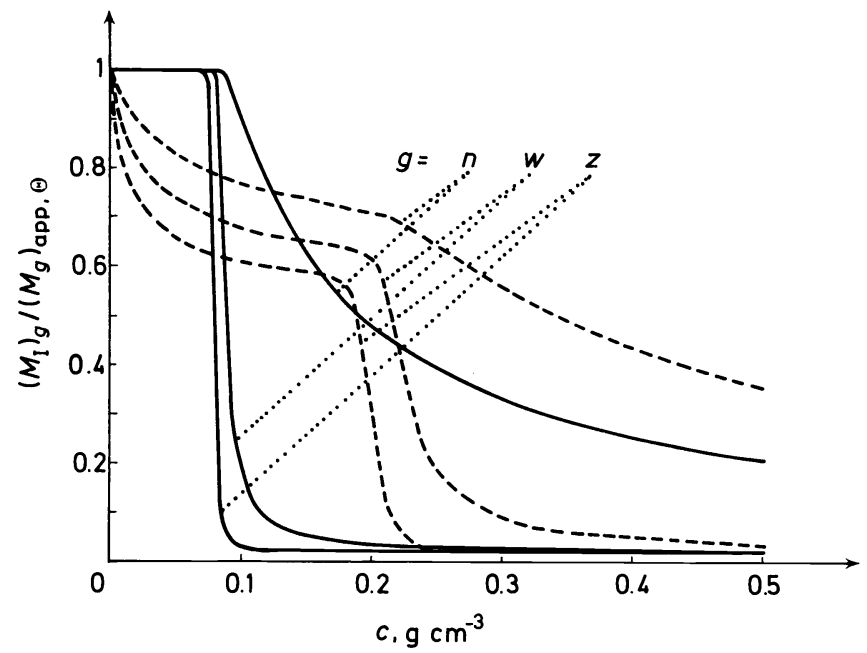

Figure 8. Concentration dependence of the reduced inverse apparent $g$-average molecular weights in the theta state for a molecule-based closed two-step association with $q=2, r=50,{ }^{n} K_{q}=$ $2500 \mathrm{dm}^{3} \mathrm{~mol}^{-1}$ and ${ }^{n} K_{r / q}=50^{50}\left(\mathrm{dm}^{3} \mathrm{~mol}^{-1}\right)^{24}$ (broken lines); the latter equilibrium constant corresponds to an apparent open equilibrium constant for the addition on a dimer of ${ }^{n} K_{0} \approx$ $3464 \mathrm{dm}^{3} \mathrm{~mol}^{-1}$. For comparison, the functions for a corresponding molecule-based closed one-step association with $r=50$ and ${ }^{n} K_{\mathrm{c}}=\left({ }^{n} K_{q}\right)^{r / q}\left({ }^{n} K_{r / q}\right)={ }^{n} K_{r}$ are given (solid lines); this corresponds to an open equilibrium constant of ${ }^{n} K_{0}=2933 \mathrm{dm}^{3} \mathrm{~mol}^{-1}$ for the addition of one unimer. $\left(M_{1}\right)_{n}=\left(M_{1}\right)_{w}=\left(M_{1}\right)_{z}=300 \mathrm{dm}^{3} \mathrm{~mol}^{-1}$

An equilibrium constant of ${ }^{n} K_{r / q}=50^{50}\left(\mathrm{dm}^{3} \mathrm{~mol}^{-1}\right)^{24}$ certainly is in the middle range of possible equilibrium constants (see Tables 6 and 7). However, model calculations with an increased equilibrium constant of ${ }^{n} K_{r / q}=5000^{50}$ and the same values for the other constants have shown that the two-step character of these curves will not be apparent within 'experimental' error. Consequently, premicellar equilibria can be observed only if the two equilibrium constants are of the same magnitude. Furthermore, the two association numbers must be sufficiently different because model calculations with $q=10, r=50,{ }^{n} K_{q}=50^{2} \mathrm{dm}^{3} \mathrm{~mol}^{-1}$ and ${ }^{n} K_{r}=50^{50}\left(\mathrm{dm}^{3} \mathrm{~mol}^{-1}\right)^{24}$ resulted also in a loss of the two-step character of these curves. 
Judging from these calculations, premicellar equilibria would seem to be detectable only in rare cases. However, many workers in the detergent field claim the existence of premicellar equilibria ${ }^{18-125}$. Most of their claims are based on the so-called Debye plots. Debye ${ }^{126,127}$ assumes basically that a unimer $/ r$-mer system can be treated as a solution of $r$-mers in a solvent composed of the unimer and the dissolving liquid, i.e. the solution is treated as a quasibinary system. The method was originally developed for light scattering measurements, but can obviously be transferred to other methods, too.

The principle of the method can be demonstrated by, e.g., light scattering measurements. The Rayleigh ratio $R$ is first plotted against the concentration $c$ in order to determine the 'critical micelle concentration' $(\mathrm{cmc})$ and the ratio $R_{\mathrm{cmc}}$ at the cmc (see Figure 9). Instead of $R$, the reduced quantity $R / K$ can be used, where $K$ is the optical constant of the light scattering equations. Following Debye's assumptions, an inverse apparent $r$-mer particle weight is then plotted against the 'true' $r$-mer concentration $(c-\mathrm{cmc})$ :

$$
\frac{1}{\left(M_{r}\right)_{\mathrm{app}}}=\frac{K(c-\mathrm{cmc})}{R-R_{\mathrm{cmc}}}=\frac{1}{\left(M_{r}\right)_{w}}+2\left(A_{2}\right)_{r}(c-\mathrm{cmc})
$$

(see also Figure 10). The intercept is supposed to give the weight average particle weight of the $r$-mer and the slope the second virial coefficient of the $r$-mer/solvent system. An upward bend at lower concentrations has been traditionally explained as due to the existence of premicellar equilibria ${ }^{118-125}$ because it corresponds to an increase of particle weights lower than $M_{r}$ with decreasing concentrations if Debye's method is correct.

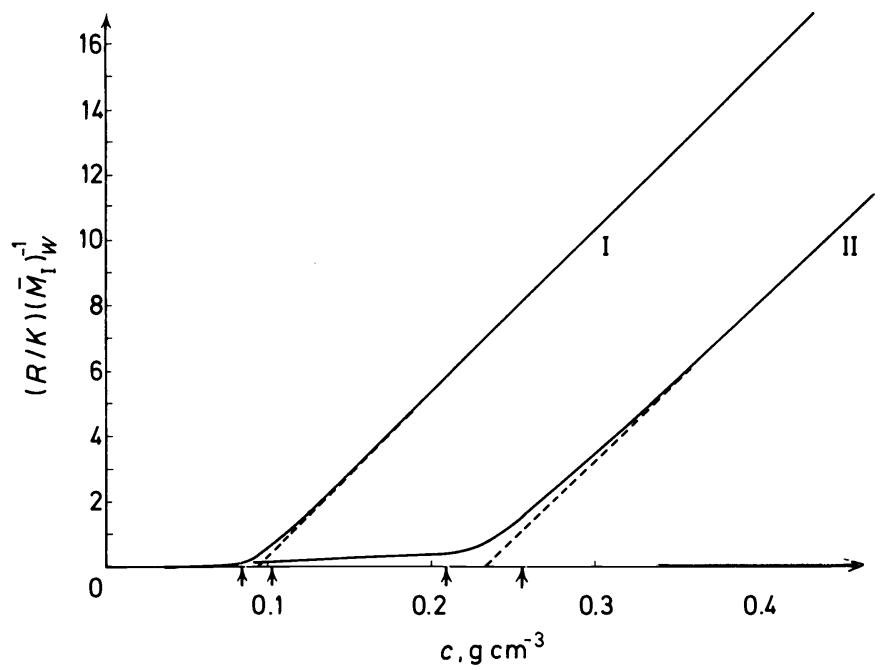

Figure 9. Concentration dependence of the normalized reduced Rayleigh ratio $(R / K)\left(M_{\mathrm{I}}\right)_{w}^{-1}$ for one-step (I) and two-step (II) molecule-based closed associations. Same assumptions as in Figure 8. 'True' critical micelle concentrations $\mathrm{cmc}$ are given by the intersection of the broken lines with the $c$-axis. Arrows indicate the 'apparent critical micelle concentrations' $\mathrm{cmc}^{\prime}$ ' which were used for the calculations leading to Figure $10 . \mathrm{cmc}^{\prime}=(1 \pm 0.1) \mathrm{cmc}$ 


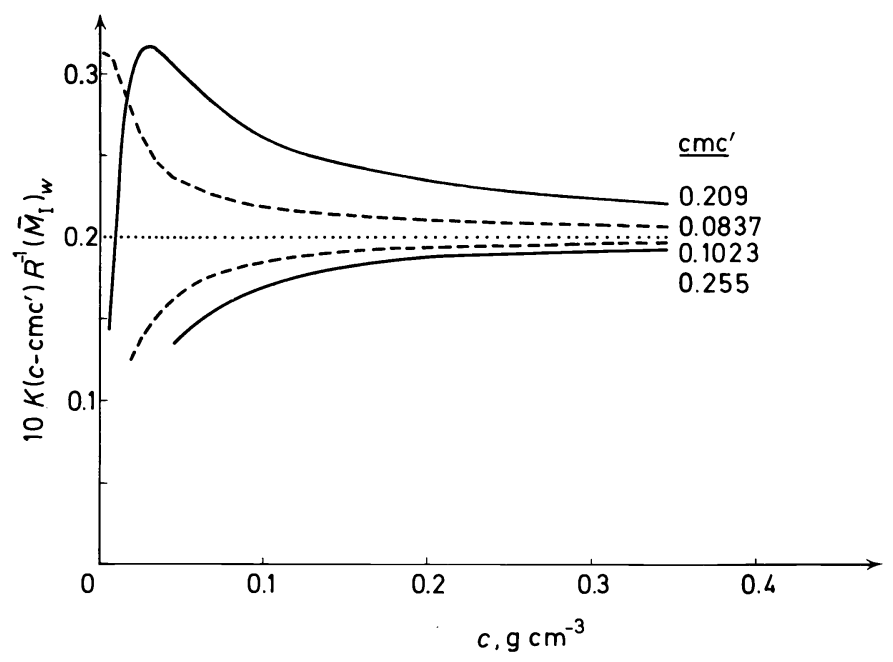

Figure 10. Normalized Debye plot for the determination of association numbers and the detection of premicellar equilibria. Solid lines indicate two-step associations, broken lines one-step associations. Same assumptions as in Figures 8 and 9. Calculations for two critical micelle concentrations $\mathrm{cmc}^{\prime}$ assuming $R_{\mathrm{cmc}^{\prime}}=0$. Dotted line corresponds to $\left(\bar{M}_{r}\right)_{w}$

As has been pointed out previously ${ }^{9}$, however, the choice of the value of cmc is very critical. If $\mathrm{cmc}$ and $R_{\mathrm{cmc}}$ values left of the asymptote line in Figure 9 are chosen, an upswing is observed even for one-step closed association (Figure 10), which may be falsely interpreted as due to premicellar equilibria. If the $\mathrm{cmc}$ and $R_{\mathrm{cmc}}$ values are chosen right from the asymptote line, a downward bend appears. We can thus conclude that an upward bend per se does not necessarily point towards the existence of premicellar equilibria.

But even if premicellar equilibria exist, they cannot be detected by the Debye plot. Again, upward and downward bends are observed, depending on the choice of cmc and $R_{\mathrm{cmc}}$. The curves for two-step equilibria are only quantitatively different from the curves for the one-step equilibria. It is easy to see why the two-step character cannot show up in a Debye plot: the determination and the choice of a cmc via an $R=f(c)$ plot wipes out all data points for premicellar equilibria because they must be by necessity at concentrations lower than the $\mathrm{cmc}$ and would thus lead to the physically unreasonable negative particle weights in the Debye treatment. Furthermore premicellar equilibria are very difficult to detect in plots like Figure 9 because they give very small $R / K$ or $R$ values at low concentrations where they are most difficult to detect.

We can thus conclude that the Debye plot cannot be used to detect premicellar equilibria because the very assumptions underlying Debye's treatment preclude the detection of premicellar equilibria even if they exist. On the other hand, the apparent existence of premicellar equilibria as shown by an upward bend in a Debye plot is most likely to result from the particular 
choice of cmc and $R_{\mathrm{cmc}}$. Premicellar equilibria, if there are any, would probably be best detected by a plot of the inverse apparent molecular weight against the concentration (see Figure 8). Even then, they may not be apparent if the association numbers $q$ and $r$ are too near to each other or if the equilibrium constants ${ }^{n} K_{q}$ and ${ }^{n} K_{r}$ are very different. The existence of premicellar equilibria thus has not been proven experimentally in a convincing manner for closed associations.

\section{ACKNOWLEDGEMENTS}

The author appreciates the help of Dr Karel Šolc, MMI, with the computer calculations and many discussions with him. The help of Professor E. T. Adams, Texas A \& M University, in searching data for protein association is gratefully acknowledged.

\section{REFERENCES}

${ }^{1}$ H. Staudinger, Ber. Dtsch. Chem. Ges. 59, 3019 (1926).

${ }^{2}$ H.-G. Elias, R. Bareiss and J. G. Watterson, Advanc. Polymer Sci. 11, 111 (1973).

${ }^{3}$ W. G. McMillan and J. E. Mayer, J. Chem. Phys. 13, 276 (1945).

${ }^{4}$ P. J. Flory and W. R. Krigbaum, J. Chem. Phys. 18, 1086 (1950).

${ }^{5}$ W. H. Stockmayer, J. Chem. Phys. 18, 58 (1950).

${ }^{6}$ J. G. Kirkwood and R. J. Goldberg, J. Chem. Phys. 18, 54 (1950).

${ }^{7}$ H. Fujita, Mathematical Theory of Sedimentation Analysis. Academic Press: New York (1962).

${ }^{8}$ H.-G. Elias and R. Bareiss, Chimia (Aarau), 21, 53 (1967).

${ }^{9}$ H.-G. Elias, in M. B. Huglin (ed.), Light Scattering from Polymer Solutions. Academic Press: London (1972).

10 D. F. Waugh, Advanc. Protein Chem. 9, 325 (1954).

${ }^{11}$ F. J. Reithel, Advanc. Protein Chem. 18, 123 (1963).

${ }^{12}$ H.-G. Elias, Intern. J. Polymeric Mat., in press; see also H.-G. Elias, in K. Solc (ed.), Order in Polymer Solutions (Midland Macromol. Monographs, Vol. 2). Gordon and Breach: London (in press).

13 E. T. Adams, Jr, Fractions, No. 3, 1 (1967).

${ }^{14}$ H. G. Bungenberg de Jong and W. A. L. Dekker, Biochem. Z. 212, 318 (1929).

15 W. Philippoff, Viskosität der Kolloide (Handbuch der Kolloidwissenschaft in Einzeldarstellungen, Bd. IX), p 333. Th. Steinkopff: Dresden-Leipzig (1942).

${ }^{16}$ P. J. Flory, J. Amer. Chem. Soc. 65, 372 (1943).

${ }^{17}$ A. R. Shultz, A. L. Bridgman, E. M. Hadsell and C. R. McCollough, J. Polymer Sci. A-2, 273 (1972).

${ }^{18}$ E. T. Adams, Jr, Proc. Nat. Acad. Sci. Wash., 51, 509 (1964).

19 V. N. Schumaker, J. Polymer Sci. 38, 343 (1959).

20 A. F. Samorin, Vysokomol. Soed. A13, 1024 (1971).

21 J. Dayantis and H. Benoit, J. Chim. Phys. 61, 773 (1964).

22 H. A. Ende, J. Polymer Sci. B3, 139 (1965).

${ }_{23}$ M. Bohdanecky, P. Kratochvil and K. Šlc, J. Polymer Sci. A3, 4153 (1965).

${ }^{24} \mathrm{~K}$. Solc, private communication.

${ }^{25}$ G. V. Schulz, Z. Phys. Chem. B43, 25 (1939).

${ }^{26}$ H. L. Frisch and J. L. Lundberg, J. Polymer Sci. 37, 123 (1959).

27 S. Ya. Frenkel, Vysokomol. Soed. 2, 731 (1960); Polymer Sci. USSR, 2, 427 (1961).

${ }^{28}$ E. Montroll, J. Amer. Chem. Soc. 63, 1215 (1941).

29 E. F. G. Herington and A. Robertson, Trans. Faraday Soc. 38, 490 (1942).

${ }^{30}$ H. H. G. Jellinek, J. Polymer Sci. 4, 399 (1949).

${ }^{31}$ G. Herdan, Nature, 163, 139 (1949).

${ }^{32}$ G. Herdan, Nature, 164, 502 (1949).

${ }^{33}$ G. Herdan, Small Particle Statistics. Elsevier: Amsterdàm (1953). 


\section{HANS-GEORG ELIAS}

${ }^{34}$ R. L. Baldwin and K. E. Van Holde, Fortschr. Hochpolymer. Forschg. 1, 451 (1960).

35 J. B. Carmichael, J. Macromol. Sci. (Chem.) A2, 1411 (1968).

36 J. G. Watterson and H.-G. Elias, J. Macromol. Sci. (Chem.) A5, 459 (1971).

${ }^{37}$ W. Kuhn, Kolloid-Z. 68, 2 (1934).

${ }^{38}$ H. Mark, in R. Sänger (ed.), Der Feste Körper. Hirzel: Leipzig (1938).

${ }^{39}$ I. Sakurada, Kasen-Koenshu, 5,33 (1940).

${ }^{40}$ R. Houwink, J. Prakt. Chem. 157, 15 (1941).

41 P. J. Flory, J. Amer. Chem. Soc. 65, 372 (1943).

42 M. Daune and L. Freund, J. Polymer Sci. 23, 115 (1957).

${ }^{43}$ T. Svedberg, Kolloid-Z., Erg.-Bd. 36, 53 (1925).

44 H. Kobayashi, J. Polymer Sci. 39, 369 (1959).

${ }^{45}$ N. Yamada and H. Matsuda, Rep. Progr. Polymer Phys. Japan, 3, 48 (1960); Kobunshi Kagaku, 18, 110, 114(1961).

46 S. Ya. Frenkel, Vysokomol. Soed. 2, 731 (1960); Polymer Sci. USSR, 2, 427 (1961).

${ }^{47}$ L. Mandelkern and P. J. Flory, J. Chem. Phys. 20, 212 (1952).

${ }^{48}$ L. Mandelkern, W. R. Krigbaum, H. A. Scheraga and P. J. Flory, J. Chem. Phys. 20, 1392 (1952).

49 H. A. Scheraga and L. Mandelkern, J. Amer. Chem. Soc. 75, 179 (1953).

${ }^{50}$ V. M. Men'shov and S. Ya. Frenkel', Vysokomol. Soed. 6, 206 (1964); Polymer Sci. USSR, 6, 236 (1964).

51 J. C. Moore, J. Polymer Sci. A2, 835 (1964).

52 J. Rehner, Ind. Engng Chem. 36, 118 (1944).

53 R. S. Spencer and R. F. Boyer, Polymer Bull. 1, 129 (1945).

${ }^{54}$ R. F. Boyer and R. Simha, in R. H. Boundy and R. F. Boyer (eds.), Styrene-its Polymers, Copolymers and Derivatives, $\mathrm{p}$ 324. Reinhold: New York (1952).

55 R. Chiang, J. Polymer Sci. 36, 91 (1959).

${ }^{56}$ R. Koningsveld and C. A. F. Tuijnman, Makromol. Chem. 38, 39 (1960).

${ }^{57} \mathrm{M}$. Wales and S. J. Rehfeld, J. Polymer Sci. 62, 179 (1962).

${ }^{58} \mathrm{M}$. Wales and S. J. Rehfeld, J. Polymer Sci. A1, 3666 (1963).

${ }^{59} \mathrm{~K}$. Solc, private communication (March 1974).

60 J. E. Hearst and W. H. Stockmayer, J. Chem. Phys. 37, 1425 (1962).

61 D. M. Crothers and B. H. Zimm, J. Mol. Biol. 12, 525 (1965).

${ }^{62}$ H. B. Gray, V. A. Bloomfield and J. E. Hearst, J. Chem. Phys. 46, 1493 (1967).

${ }^{63}$ P. Sharp and V. A. Bloomfield, J. Chem. Phys. 48, 2149 (1968).

${ }^{64}$ K. E. Reinert, Studia Biophysica, 10, 73 (1968).

${ }^{65}$ K. E. Reinert, Biopolymers, 10, 275 (1971).

${ }^{66}$ N. Yamada, H. Matsuda and H. Nakamura, Rep. Progr. Polymer Phys. Japan, 4, 81 (1961).

${ }^{67}$ K. G. Berdnikova, G. V. Tarasova, V. S. Skazka, N. A. Nikitin and G. V. Dyuzhev, Vysokomol. Soed. 6, 2057 (1964); Polymer Sci. USSR, 6, 2280 (1965).

${ }^{68}$ H.-G. Elias, Makromol. Chem. 122, 264 (1969).

${ }^{69}$ H. G. Elias and R. Bareiss, J. Macromol. Sci. (Chem.) A1, 1377 (1967).

${ }^{70}$ A. Kotera, T. Saito and K. Takemura, IUPAC Congress Boston 1971, Macromolecular Preprints, p 1029.

${ }^{71}$ H. Sueoka, Proc. Nat. Acad. Sci. Wash. 45, 1480 (1959).

${ }^{72}$ T. Homma and H. Fujita, J. Appl. Polymer Sci. 9, 1701 (1965).

${ }^{73}$ H. W. McCormick, J. Polymer Sci. 36, 341 (1959).

${ }^{74}$ V. Souček, Makromol. Chem. 99, 287 (1966).

${ }^{75}$ M. Kalfus and J. Mitus, J. Polymer Sci. (A-1) 4, 953 (1966).

${ }^{76}$ M. Kalfus and H.-G. Elias, J. Macromol. Sci. (Chem.) A1, 955 (1967).

${ }^{77}$ H.-G. Elias and M. Kalfus, Makromol. Chem. 105, 95 (1967).

${ }^{78}$ V. Soucek, Coll. Czech. Chem. Commun. 33, 1962 (1968).

79 H. Baumann, J. Polymer Sci. B3, 1069 (1965).

${ }^{80}$ W. Sutter and A. Kuppel, Makromol. Chem. 149, 271 (1971).

${ }^{81}$ R. E. Bareiss, Makromol. Chem. 170, 251 (1973).

82 R. Koningsveld and C. A. F. Tuijnman, Makromol. Chem. 38, 39 (1960).

${ }^{83}$ R. E. Bareiss and J. Bellido (in preparation).

${ }^{84}$ R. E. Bareiss, in J. Brandrup and E. H. Immergut (eds.), Polymer Handbook, 2nd ed. Interscience: New York (1974).

${ }^{85}$ H.-G. Elias and F. Patat, Makromol. Chem. 25, 13 (1957). 
${ }^{86}$ A. Münster and H. Diener, IUPAC Internat. Symp. Makromolekiile, Wiesbaden 1959, Preprint II B 5.

${ }^{87}$ W. Cooper, G. Vaughan, D. E. Eaves and R. W. Madden, J. Polymer Sci. 50, 159 (1961).

${ }^{88}$ R. Schumacher and H.-G. Elias, Makromol. Chem. 76, 12 (1964).

89 E. F. Casassa, Polymer (London), 1, 169 (1960).

90 W. R. Krigbaum and P. J. Flory, J. Amer. Chem. Soc. 75, 1775 (1953).

91 V. V. Varadaiah and V. S. R. Rao, J. Polymer Sci. 50, 31 (1961).

92 T. Kato, K. Miyaso and M. Nagesawa, J. Phys. Chem. 72, 2161 (1968).

93 H. Utiyama, N. Tagata and M. Kurata, J. Phys. Chem. 73, 1448 (1969).

94 T. P. Wallace and E. F. Casassa, ACS Polymer Preprints, 11, 136 (1970).

95 H.-G. Elias, J. Bellido and R. Bareiss, Makromol. Chem. 176, 439 (1975).

$96 \mathrm{~W}$. Gobush, private communication.

97 H.-G. Elias and K. Solc, Makromol. Chem. 176, 395 (1975).

98 K. Solc and H.-G. Elias, J. Polymer Sci. (Phys.) 11, 137 (1973).

99 P. J. Flory, Principles of Polymer Chemistry, p 331. Cornell University Press: Ithaca, N.Y. (1953).

100 H.-G. Elias, Makromoleküle, 2nd ed., p 579. Hüthig and Wepf: Basel (1972).

101 J. Gerber and H.-G. Elias, Makromol. Chem. 112, 142 (1968).

102 H.-G. Elias and J. Gerber, Makromol. Chem. 112, 122 (1968).

103 H. R. Lässer and H.-G. Elias, Kolloid-Z. Z. Polym. 250, 58 (1972).

104 S. Ikeda and K. Kakiuchi, J. Colloid Interf. Sci. 23, 134 (1967).

105 H.-R. Lässer and H.-G. Elias, Kolloid-Z. Z. Polym. 250, 44 (1972).

106 U. Kammer and H.-G. Elias, Kolloid-Z. Z. Polym. 250, 344 (1972).

107 E. T. Adams, Jr, and M. S. Lewis, Biochemistry, 7, 1044 (1968).

${ }^{108}$ L.-H. Tang and E. T. Adams, Jr, Arch. Biochem. Biophys. 157, 520 (1973).

109 D. A. Albright and J. W. Williams, Biochemistry, 7, 67 (1968).

110 J. N. Timasheff and R. Townend, J. Amer. Chem. Soc. 83, 470 (1961).

111 J. L. Sarquis and E. T. Adams, Jr, Arch. Biochem. Biophys. (in press)

112 P. D. Jeffrey and J. H. Coates, Biochemistry, 5, 489 (1966).

113 P. W. Chun, S. J. Kim, J. D. Williams, W. T. Cope, L. H. Tang and E. T. Adams, Jr, Biopolymers, 11, 197 (1972); value re-evaluated from ref. 114.

$114 \mathrm{H}$. Eisenberg and G. Tomkins, J. Mol. Biol. 31, 37 (1968).

115 A. H. Pekar and B. H. Frank, Biochemistry, 11, 4013 (1972).

116 D. B. S. Miller, G. E. Willick, R. F. Steiner and V. Frattali, J. Biol. Chem. 244, 287 (1969).

117 J. B. Harry and R. F. Steiner, Biochemistry, 8, 5060 (1969).

118 L. M. Kushner and W. D. Hubbard, J. Phys. Chem. 58, 1163 (1954).

119 L. M. Kushner, W. D. Hubbard and A. S. Doan, J. Phys. Chem. 61, 371 (1957).

120 R. R. Balmbra, J. S. Clunie, J. M. Corkill and J. F. Goodman, Trans. Faraday Soc. 58, 1661 (1962).

121 R. R. Balmbra, J. S. Clunie, J. M. Corkill and J. F. Goodman, Trans. Faraday Soc. 60, 979 (1964).

122 P. H. Elworthy and C. B. Macfarlane, J. Chem. Soc. (London), 907 (1963).

123 J. M. Corkill, J. F. Goodman and T. Walker, Trans. Faraday Soc. 63, 759 (1967).

124 D. Attwood, J. Phys. Chem. 72, 339 (1968).

125 P. Mukerjee, J. Phys. Chem. 76, 565 (1972).

126 P. Debye, J. Colloid Sci. 3, 407 (1948).

127 P. Debye, Ann. NY Acad. Sci. 51, 575 (1949). 